MATHEMATICS OF COMPUTATION

Volume 69, Number 229, Pages 197-235

S $0025-5718(99) 01147-3$

Article electronically published on August 19, 1999

\title{
FROM FORMAL NUMERICAL SOLUTIONS OF ELLIPTIC PDE'S TO THE TRUE ONES
}

\author{
Z. WIENER AND Y. YOMDIN
}

\begin{abstract}
We propose a discretization scheme for a numerical solution of elliptic PDE's, based on local representation of functions, by their Taylor polynomials (jets). This scheme utilizes jet calculus to provide a very high order of accuracy for a relatively small number of unknowns involved.
\end{abstract}

\section{INTRODUCTION}

This work is devoted to an introduction and a preliminary investigation of a discretization method, based on representation of functions by their Taylor polynomials on a certain grid.

We tried to achieve three main goals:

1. A general description of the proposed discretization and the numerical scheme based on it, and discussion of its main features, advantages and restrictions. Initial theoretical investigation of this general scheme.

2. Construction of a high-accuracy scheme for a special example of the Laplace and Poisson equations. Investigation of the discretization error and a preliminary experimental testing of its accuracy and stability.

3. Description of a multiscale solution scheme, which allows for a significant reduction of the stiffness of the equation to be solved, and a preliminary experimental investigation.

Our discretization scheme for solving elliptic PDE's is based on Kolmogorov's optimal representation of smooth functions on the one hand, and on Gromov's approach to solving PDE's on the other (see [5], [2]). This method has been proposed in a general form in [11] and partially implemented in [3], [4]. It consists of several steps.

1. All the functions involved (known and unknown) are represented by their Taylor polynomials of a fixed degree $k$ at all the nodes of a certain fixed grid.

2. The Taylor polynomials for the unknown functions (whose coefficients form the basic set of the unknowns) are a priori parametrized to satisfy locally the PDE to be solved. For example, for the equation $\Delta u=0$, harmonic polynomials are used at each grid point. The boundary conditions are incorporated into the parametrization of the Taylor polynomials at the grid points near the boundary. However, for the parameter values, picked at random, the above Taylor polynomials satisfying the equation do not agree with one another. In Gromov's terminology [2], they form a nonholonomic section of a differential relation and do not represent a true

Received by the editor October 14, 1994 and, in revised form, May 23, 1997.

1991 Mathematics Subject Classification. Primary 65N06, 65N15, 35J05.

(C)1999 American Mathematical Society 
solution of the equation. Thus the solution process consists of finding the values of the unknown parameters which minimize the discrepancy between the neighboring Taylor polynomials. This approach can be considered to be a discretized realization of Gromov's $h$-homotopy. In such a terminology the standard methods use a true function which approximately satisfies the differential relation. Our method uses objects which are not true functions but satisfy the differential relation exactly.

3 . We implement the last stage of the solution as a certain relaxation procedure where the Taylor polynomial at each node is corrected according to the neighboring ones. The mere presence of several Taylor coefficients at each node (instead of the only one in standard schemes) allows one to find relaxation coefficients which "cancel" the discretization error of the solution up to an order $m$ which is much higher than $k$. For example, for $\Delta u=0$ for the second degree Taylor polynomials, for an internal node we get the discretization error of order $h^{10}$, where $h$ is the step of the grid.

4. At the previous stage we got at each grid point a Taylor polynomial of degree $k$ which agrees with the Taylor polynomial of the true solution at this point up to order $m>k$. It turns out that from this data one can usually reconstruct at each grid point the $m$-th degree Taylor polynomial of the true solution, with the same accuracy. For this reconstruction, the same neighboring nodes as those for relaxation, are used. Figure 1 gives a pictorial explanation of the method.

Let us now discuss in more detail the reasons behind the choice of this scheme and some of its features.

The question of an optimal $\varepsilon$-representation of smooth functions has been investigated by Kolmogorov in his work on $\varepsilon$-entropy of functional classes [5]. It was shown that asymptotically, the best way to memorize a $C^{k}$-function up to accuracy $\varepsilon>0$ is to store the coefficients of its $k$-th order Taylor polynomials at each point of some grid of size $h=O\left(\varepsilon^{1 /(k+1)}\right)$.

The following point implied by the Kolmogorov representation is very important in our approach: We assume that a required accuracy or tolerance $\varepsilon>0$ is given from the very beginning. We require the discretized data to represent the "true" function up to this accuracy in a $C^{k}$-norm. But we do not require the discretized data to be itself a $C^{k}$-function. In particular, the Taylor polynomials at the neighboring grid points may disagree up to $\varepsilon$.

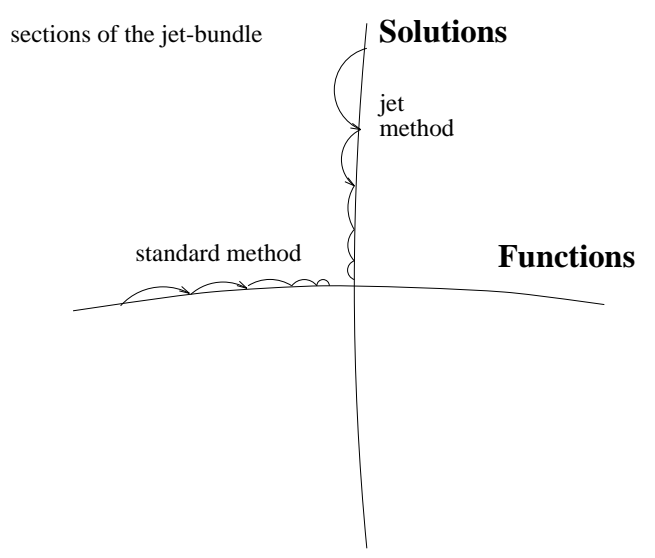

FiguRE 1 
This makes our representation very flexible. In particular, we make no effort to subdivide the domain into pieces where each of the polynomials is used or to adjust their values (and/or the values of their derivatives) at the boundaries of such pieces.

Notice that this subdivision and adjustment is by far the most complicated part in many high-order algorithms. Moreover, it introduces into the solution process a rigid combinatorial-geometric structure, which has nothing to do with the equation to be solved.

An important requirement for a discretization scheme used in analytic computations is that any analytic operation or functional, applied to a given function, must be expressible (up to accuracy $\varepsilon$ ) in terms of a discretization of this function. Our scheme satisfies this requirement (see [11]).

In some applications, such as computer graphics and engineering, it is important to be able to produce ultimately a truly continuous or smooth function from the discretized data. In our scheme this can be done using a partition of unity.

The absence of adjustment of neighboring polynomials makes the scheme described relatively insensitive to the precise geometry of the grid. This geometry is involved only in the computation of the optimal relaxation coefficients (see subsections 4.1 and 5.1).

Also near the boundary no adjustment of the grid geometry is necessary. Using the calculus of Taylor polynomials, we incorporate the boundary data into the structure of the Taylor polynomials at the grid points near the boundary with the maximal accuracy required (see subsection 3.2).

The following two remarks explain why we can expect the suggested scheme to provide a very high order of a discretization error for a relatively small number of unknowns.

1. We use jets at each grid point which satisfy the initial differential equation. This strongly reduces the number of free parameters. For example, for two independent variables, general jets of degree $k$, contain $\frac{(k+1)(k+2)}{2}$ parameters. Jets of degree $k$, satisfying a linear PDE with constant coefficients of order 2 , have $2 k+1$ free parameters (see subsection 3.2).

Notice that the requirement for local representing elements to satisfy the initial differential equation is usually not compatible with the precise adjustment of values and derivatives for neighboring elements (since the last requirement leads to elements with compact support).

2. We find a relaxation scheme (i.e., equations relating a Taylor polynomial at each grid point with its neighboring polynomials) which provides a highest order discretization error. Since we spend no degrees of freedom to provide boundary adjustment of neighboring polynomials, enough degrees of freedom remain to "cancel" the Taylor coefficients in a discretization error up to a high order. The following rough computation shows what order of accuracy can be expected.

For a second-order equation and jets of degree $k$ we have $2 k+1$ parameters at each grid point. Thus at four nearest neighboring points, we have $8 k+4$ parameters. Assuming that the systems which arise are solvable, we can reconstruct (at the central point) a jet of degree $4 k$ satisfying the equation, or we can cancel the Taylor terms in the discretization error up to the same degree. In subsection 4.1 we describe this procedure in detail, showing in particular that for $\Delta u=0$, for a regular grid and $k=2$, the corresponding equations are solvable up to degree 10 because of a symmetry in the problem. 
Notice that wider neighborhood stencils can be used in the relaxation procedure. However, geometrically compact schemes have important computational advantages.

The aim of this work is mostly to describe the Taylor polynomial discretization scheme from the point of view of its structure, and the discretization errors produced. However, there is an important stability problem to be addressed. In fact, this problem is encountered by any numerical method involving an explicit treatment of high-order derivatives: such schemes inevitably contain coefficients proportional to high negative degrees of the grid size which makes them very sensitive to a numerical noise.

We have investigated two basic approaches which allow us to overcome this difficulty. The first one is described in detail in subsection 4.1. It is based on the fact that we can work with the triangular relaxation schemes in which derivatives of a certain order are influenced only by the derivatives of the same and higher orders of the neighboring jets. The accuracy of such schemes is, of course, lower than for full ones. Triangular schemes can be split into a chain of successively solved stable schemes, starting with the highest order derivatives.

The second approach is based on the full schemes, which provide the highest order of a discretization error, but cannot be split in any obvious way. Here the stability problem can be solved by a multiscale approach starting with a low order scheme, and then solving at each step a one order higher residual problem (properly rescaled). Below we describe this multiscale procedure in detail and give some initial numerical results.

A similar discretization scheme can be used for other types of equations. In particular, some initial results, concerning the discretization error and stability of the above type schemes for parabolic equations are given in [1]. Numerical approaches, based on Taylor approximation, are quite popular in solving ordinary differential equations (see [8] for example). To our best knowledge, such approaches are much less investigated in PDE's, however (see [8] and [12]), where somewhat similar methods are proposed.

The authors would like to thank the referee for useful suggestions which helped us to improve the presentation.

\section{BASIC FACTS AND DEFINITIONS}

2.1. Jets and jet-functions. We say that two real-valued functions $f$ and $g$ both defined in some neighborhood of a point $z \in \mathbb{R}^{n}$ are $k$ equivalent if they coincide at this point up to derivatives of order $k$. Identifying functions with respect to this equivalence relation, we get the space of $k$-jets at the point $z$ denoted further as $\operatorname{Jet}_{k}^{z}$. We denote as jet ${ }_{k}^{z} f$ the $k$-th order jet of a function $f$ at a point $z \in \mathbb{R}^{n}$. The dimension of $\operatorname{Jet}_{k}^{z}$ is

$$
1+n+\frac{n(n+1)}{2}+\cdots+\frac{(n+k-1) !}{(n-1) ! k !}=\frac{(n+k) !}{n ! k !} .
$$

We denote by $\mathcal{B}_{k}^{z}$ the set of all multi-indexes $\alpha=\left(\alpha_{1}, \alpha_{2}, \cdots, \alpha_{n}\right)$ describing all jets of order $k$ at a point $z$.

Consider a compact set $G \subset \mathbb{R}^{n}$, and a bundle with the fiber $\operatorname{Jet}_{k}^{z}$ at each point $z \in G$. We denote the set of all sections $U(z)$ of this bundle by $\mathrm{JEF}_{k}^{G}$ and call them jet-functions. They form a finite dimensional linear space in the case when $k<\infty$ and the set $G$ is finite; otherwise, the space $\mathrm{JEF}_{k}^{G}$ is infinite dimensional. 
Arithmetic operations on $\mathrm{JEF}_{k}^{G}$ are naturally defined pointwise. Each jet has a polynomial representative, so we can easily define a shift operation just by expanding the same polynomial at another point. In fact the shift operator is another form of the Taylor formula written in the form of a linear operator on the Jet space $S^{z, \tilde{z}}: \operatorname{Jet}_{k}^{z} \rightarrow \operatorname{Jet}_{k}^{\tilde{z}}$. For example a shift of a 2 -jet defined at $(0,0) \in \mathbb{R}^{2}$ to a point $(x, y)$ is

$$
S^{(0,0),(x, y)}\left(\begin{array}{c}
U_{00} \\
U_{10} \\
U_{01} \\
U_{20} \\
U_{11} \\
U_{02}
\end{array}\right)=\left(\begin{array}{c}
U_{00}+U_{10} x+U_{01} y+U_{20} \frac{x^{2}}{2}+U_{11} x y+U_{02} \frac{y^{2}}{2} \\
U_{10}+U_{20} x+U_{11} y \\
U_{01}+U_{11} x+U_{02} y \\
U_{20} \\
U_{11} \\
U_{02}
\end{array}\right) .
$$

The corresponding operator is given by the following matrix:

$$
S^{(0,0),(x, y)}=\left(\begin{array}{cccccc}
1 & x & y & \frac{x^{2}}{2} & x y & \frac{y^{2}}{2} \\
0 & 1 & 0 & x & 0 & 0 \\
0 & 0 & 1 & 0 & y & 0 \\
0 & 0 & 0 & 1 & 0 & 0 \\
0 & 0 & 0 & 0 & 1 & 0 \\
0 & 0 & 0 & 0 & 0 & 1
\end{array}\right)=\exp \left(\begin{array}{cccccc}
0 & x & y & 0 & 0 & 0 \\
0 & 0 & 0 & x & y & 0 \\
0 & 0 & 0 & 0 & x & y \\
0 & 0 & 0 & 0 & 0 & 0 \\
0 & 0 & 0 & 0 & 0 & 0 \\
0 & 0 & 0 & 0 & 0 & 0
\end{array}\right) .
$$

Denote the Taylor expansion of a jet $U$ at a point $a \in G$ by

$$
T_{k}^{a} U(z)=\sum_{|\alpha| \leq k} \frac{(z-a)^{\alpha}}{\alpha !} U_{\alpha}^{a} .
$$

Another important operation defined on jets is composition (substitution) which corresponds to a polynomial change of coordinates. So given a $k$-jet $U: \mathbb{R}^{n} \rightarrow \mathbb{R}^{m}$ and a $k$-jet $V: \mathbb{R}^{l} \rightarrow \mathbb{R}^{n}$, one can define $U \circ V$ as a composition of polynomial mappings with only terms up to order $k$ preserved.

2.2. Extendible jet-functions. Any function $f \in C^{k}$ has its representative in $\mathrm{JEF}_{k}^{G}$, namely the following section: $z \mapsto \operatorname{jet}_{k}^{z} f$. Such a section we call holonomic following [2]. For some $G$ the space $\mathrm{JEF}_{k}^{G}$ contains also nonholonomic sections. For example in case $G=[0,1] \subset \mathbb{R}, k=1$, the section $U_{0}(z) \equiv 1, U_{1}(z) \equiv 1$ cannot be represented as a true function.

There exists a well-known criterion for a section of the JEF bundle to be holonomic. It can be formulated geometrically as a tangency to the Cartan distribution [9], or algebraically [6] through the Whitney functional.

The following Whitney's theorem [10] describes jet-functions which are extendible from a compact set $G \subset \mathbb{R}^{n}$ to the whole space.

Whitney theorem. If $U \in \mathrm{JEF}_{k}^{G}$ and for any $x, y \in G, z \in \mathbb{R}^{n}$

$$
\left|T_{k}^{x} U(z)-T_{k}^{y} U(z)\right| \leq \rho(|x-y|) \cdot\left(|x-z|^{k}+|y-z|^{k}\right),
$$

where the function $\rho$ is a modulus of continuity, ${ }^{1}$ then there exists a function $u$ ( $k$ times differentiable in $G$ and smooth outside) defined on a ball $B_{r}$ of an arbitrary big radius $r$ and constants $C_{\alpha}$ such that

\footnotetext{
${ }^{1}$ an increasing continuous convex function vanishing at zero
} 
1) for every $|\alpha| \leq k, x \in G, z \in B_{r}$

$$
\left|D^{\alpha} u(z)-D^{\alpha} T_{k}^{x} U(z)\right| \leq C_{\alpha} \rho(|z-x|) \cdot|z-x|^{k-|\alpha|} ;
$$

2) for every $|\alpha|>k, z \in B_{r} \backslash G$

$$
\left|D^{\alpha} u(z)\right| \leq C_{\alpha} \rho(\operatorname{dist}(z, G)) \cdot \operatorname{dist}(z, G)^{k-|\alpha|} .
$$

The proof of this theorem is given in [6], for example.

2.3. Discrete version of Whitney theorem. In the situation we have in mind, the support set $G$ is a finite grid. We assume $G$ to be $c_{1} h$-quasiregular, which means that the union of balls of radius $h$ centered at all grid nodes covers the whole set $\Omega$, and the distance between any two points of the grid is not smaller than $c_{1} h$ (for some constant $c_{1}>0$ ). We assume that for each node $z \in G$ a set of its neighbors $N(z) \subset G$ is defined which forms a stencil for the discretization scheme developed below. We also assume that these stencils are chosen in such a way that there exists a constant $c_{2}>0$ such that for any node $z$ and $\tilde{z} \in N(z)$ the distance between $z$ and $\tilde{z}$ does not exceed $c_{2} h$.

Introduce a norm on the space $\mathrm{Jet}_{k}$ :

$$
\|U\|=\max _{\alpha \in \mathcal{B}_{k}} h^{|\alpha|-k-1} \cdot\left|U_{\alpha}\right| .
$$

Define the full Whitney functional as

$$
\mathrm{WF}_{\text {full }}(U)=\max _{z_{j}, z_{l} \in G}\left\|S^{z_{j}, z_{l}} U^{z_{j}}-U^{z_{l}}\right\| .
$$

This functional is not a norm because it vanishes on global polynomials of degree $\leq k$ but it measures in some sense how far a given jet-function is from the class of smooth functions, or what the degree of incoherence of jets is at different nodes. But in fact it is very similar to the modulus of continuity which is used in the Whitney theorem.

Definition 2.3.1. Let $U_{k}$ be a jet-function defined on a quasiregular grid $G$ and $f$ a $k$-times differentiable function. We say that $f$ is $c$-represented by $U$ if for any $z_{j} \in G$, and any $\alpha \in \mathcal{B}_{k}^{z_{j}}$

$$
\max _{\left|z_{j}-z\right|<h}\left\|\operatorname{jet}_{k}^{z} f-S^{z_{j}, z} U^{z_{j}}\right\|<c .
$$

In other words this means that the function $f$ together with all its derivatives up to order $k$ lies in a tube of radius $c$ around the given jet-function $U$ with weights $h^{k+1-|\alpha|}$ coming from the Taylor formula (see Figure 2.3).

For any finite set $G$ any jet-function defined on it can be extended to a smooth function defined in the whole space. But it is natural to ask for an appropriate control on derivatives growth in intermediate points.

Discrete Whitney theorem. There exists a constant $c$ (depending on the grid) such that for any jet-function $U$ defined on an h-quasiregular grid $G$ with $\mathrm{WF}(U)=$ $M$ there exists a $k+1$ times smooth function $u$ defined in an open ball $B_{r}$ containing $G$ in which $u$ is $c M$-represented by $U$ and $\left\|D^{k+1} u\right\|<c M$. 


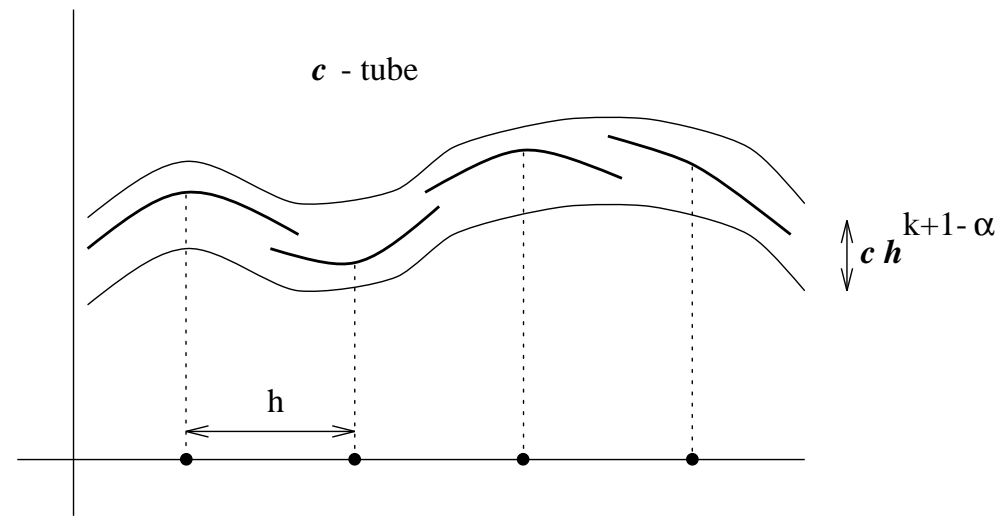

FiguRE 2.3

The proof of this theorem is very similar to the proof of the original version of the Whitney theorem (see [6], [10]). We do not provide it here. It is important for us that the full Whitney functional in the case of a discrete grid can be replaced by its short version (without significant changes in the proof)

$$
\mathrm{WF}_{\text {short }}(U)=\max _{z_{j} \in G} \max _{z_{l} \in N\left(z_{j}\right)}\left\|S^{z_{j}, z_{l}} U^{z_{j}}-U^{z_{l}}\right\| .
$$

This form of the functional is local and it requires much less computational effort. From now on we use this short version of the Whitney functional.

Remark. From the proof of the Whitney theorem it follows that one can always choose a smooth function $u$ such that its jets of order $k$ coincide exactly with given jets at all nodes of the grid.

\section{DESCRIPTION OF THE METHOD}

3.1. Discretization scheme of the same order as the degree of jets. Here we present a straightforward jet discretization scheme for elliptic equations (see [3], [4]).

Definition 3.1.1. A jet $U^{z} \in \operatorname{Jet}_{k}^{z}$ satisfies the differential equation $L u=0$ (here $L$ is a differential operator of order $m$ ) if its coordinates interpreted as partial derivatives, satisfy the equations obtained from $L u=0$ by differentiation up to the order $k-m$.

We denote the subset of jets satisfying $L u=0{\text { by } \mathrm{DE}_{k}^{z} \subset \mathrm{Jet}_{k}^{z} \text {. Clearly, jet }}_{k} u(z)$ of any function $u$ satisfying $L u=0$ belongs to $\mathrm{DE}_{k}^{z}$.

For a linear equation, $\mathrm{DE}_{k}^{z}$ is an affine subspace of $\mathrm{Jet}_{k}^{z}$. For a nonlinear equation of order $m$, this is an algebraic subset of $\operatorname{Jet}_{k}^{z}$. For any $k>m, \mathrm{DE}_{k}^{z}$ can be represented as an affine bundle over $\mathrm{DE}_{k-1}^{z}$ since the highest derivatives enter linearly in any equation obtained by differentiating the original one [2].

Consider some examples. 


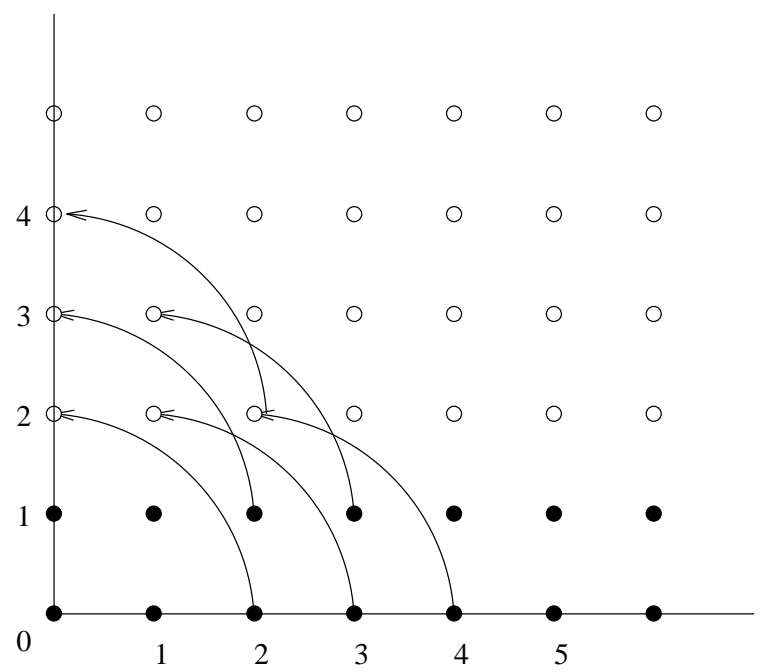

FiguRE 3.1.1

Example 3.1.1. The equation is $L u=u_{t}+u \cdot u_{x}=0, k=2$. The jet $U=$ $\left(U_{00}, U_{10}, U_{01}, U_{20}, U_{11}, U_{02}\right)^{T}$ must satisfy

$$
\begin{aligned}
& u_{t}+u \cdot u_{x}=0, \\
& u_{t t}+u_{t} \cdot u_{x}+u \cdot u_{x t}=0, \\
& u_{t x}+u_{x}^{2}+u \cdot u_{x x}=0,
\end{aligned}
$$

that is,

$$
\left\{\begin{array}{l}
U_{10}+U_{00} \cdot U_{01}=0 \\
U_{20}+U_{10} \cdot U_{01}+U_{00} \cdot U_{11}=0 \\
U_{11}+U_{01}^{2}+U_{00} \cdot U_{02}=0 .
\end{array}\right.
$$

Notice that we can parametrize the algebraic manifold $\mathrm{DE}_{k}$, taking $U_{00}, U_{01}$ and $U_{02}$ as parameters and in fact the manifold does not depend on a point $z$ :

$$
\begin{aligned}
& U_{10}=-U_{00} \cdot U_{01}, \\
& U_{11}=-U_{01}^{2}-U_{00} \cdot U_{02}, \\
& U_{20}=U_{00} \cdot U_{01}^{2}+U_{00} \cdot\left(U_{01}^{2}+U_{00} \cdot U_{02}\right) .
\end{aligned}
$$

Example 3.1.2. The example is $\Delta u=0$. Then $U_{00}, U_{10}, U_{01}, U_{20}, U_{11}, U_{30}, U_{21}, \ldots$ can be used as coordinates on the manifold of solutions and we can express any derivative through them:

$$
\begin{aligned}
& U_{02}=-U_{20}, U_{12}=-U_{30}, U_{03}=-U_{21}, \\
& U_{22}=-U_{40}, U_{13}=-U_{31}, U_{04}=U_{40} \ldots
\end{aligned}
$$

Representing the nonzero monomials $U_{\alpha \beta}$ by the integer points on the $(\alpha, \beta)$ plane (the Newton diagram) we get Figure 3.1.1. 


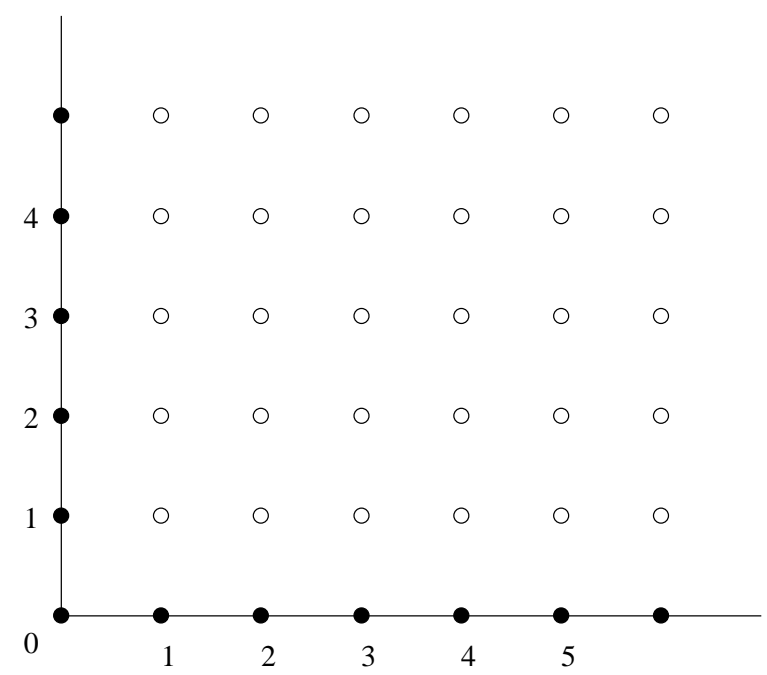

FiguRE 3.1 .2

Example 3.1.3. The example is $\Delta u=f$. The same set as in Example 3.1 .2 can be used as coordinates but the formulae expressing other derivatives take the following form:

$$
U_{02}=-U_{20}+f, U_{12}=-U_{30}+f_{x}, U_{03}=-U_{21}+f_{y}, U_{22}=-U_{40}+f_{x x}, \ldots
$$

Example 3.1.4. The example is $u_{x y}=0$. The manifold of solutions is spanned by $U_{00}, U_{10}, U_{01}, U_{20}, U_{02}, \ldots$, which reflects the fact that solutions of this equation have a form of a sum of a function of $x$ plus a function of $y$ (see Figure 3.1.2).

3.2. Linear equations. If the operator $L$ has the form $\sum_{|\alpha| \leq m} a_{\alpha}(z) \frac{\partial^{\alpha}}{\partial z^{\alpha}}$, then all the consequences are also linear - so the DE is an affine subspace of Jet.

For a linear equation with constant coefficients, we can give a simple geometric interpretation of its jet-solutions. Each operator $a_{\alpha} \frac{\partial^{\alpha}}{\partial z^{\alpha}}$ maps a monomial from position $\gamma$ on the Newton picture to the position $\gamma-\alpha$. This means that for any multi-index $\beta,|\beta| \leq k-m$ we obtain the following equation:

$$
\sum_{|\alpha| \leq m} a_{\alpha} \cdot u_{\alpha+\beta}=0
$$

Lemma 3.2.1. All the equations (3.2.1) are linearly independent.

Proof. We give the proof of the two-dimensional case by induction in $|\beta|$. Assume that for $|\beta| \leq s<k-m$, the equations are linearly independent. Consider the equations with $|\beta|=s+1$. Clearly each of these new equations contains unknowns $u_{\gamma}$ with $|\gamma|=s+1+m$ which did not appear in the equations with $|\beta| \leq s$. So the equations (3.2.1) are linearly independent.

Corollary. If $L$ is a linear differential operator of degree $m$ with constant coeffcients, then $\operatorname{dim} \mathrm{DE}_{k}=\operatorname{dim} \mathrm{Jet}_{k}-\operatorname{dim} \mathrm{Jet}_{k-m}$.

In particular, for $n=2, m=2$,

$$
\operatorname{dim} \mathrm{DE}_{k}=\frac{(k+1)(k+2)}{2}-\frac{(k-1) k}{2}=2 k+1 .
$$


For linear differential operators with variable coefficients, $\operatorname{dim} \mathrm{DE}_{k}^{z}$ can jump from point to point. A simple example is $x u^{\prime}(x)-u(x)=0$, where $\operatorname{dim} \mathrm{DE}_{k}^{x}=1$ for $x \neq 0$ and $\operatorname{dim} \mathrm{DE}_{k}^{0}=0$.

We will always assume that a parametrization of the subspace $\mathrm{DE}_{k}^{z}$ is chosen, in such a way that the free parameters are some of the coordinates $U_{\gamma}$ of Jet ${ }_{k}^{z}$, $\gamma \in \overline{\mathcal{B}}_{k}^{z} \subseteq \mathcal{B}_{k}^{z}$. For linear equations with constant coefficients one can usually choose these "free" parameters in a simple, natural way, due to a simple geometric structure of the equations, defining $\mathrm{DE}_{k}$.

For the Laplace operator, as was shown before, one can choose the set

$$
\left\{U_{p 0}, U_{q 1}, 0 \leq p \leq k, 0 \leq q \leq k-1\right\}
$$

as free coordinates, then all other coefficients can be easily found through the differential equation.

For the equation $u_{x y}=0$, we can take the set $\left\{U_{p 0}, U_{0 p} 0 \leq p \leq k\right\}$ as independent coefficients.

3.3. Boundary conditions. Now assume that the point $z \in \Omega$ belongs to a small neighborhood of the boundary $\Gamma$. We will show how to impose boundary conditions $\left.u\right|_{\Gamma}=\varphi$ on the space $\mathrm{Jet}_{k}^{z}$. Other types of boundary conditions can be discretized in a similar way. Consider a node $z \in G$ which is close to $\Gamma$. Near this point we can represent the boundary as a graph of a function depending on one variable, say $x$, by the implicit function theorem. Then $\Gamma$ is locally a graph $y=g(x)$. Choose a point $\bar{z}$ close to $z$ on $\Gamma$ and expand $g$ in Taylor series at $\bar{z}$ (see Figure 3.3.1). The boundary condition then takes the form

$$
U^{z} \circ \operatorname{jet}_{k}^{\bar{z}}(g)=\operatorname{jet}_{k}^{\bar{z}}(\varphi)
$$

that is, a composition of the jet at $z$ with the equation of the boundary should coincide with a jet of boundary values.

Thus we obtain a manifold $\mathrm{Sol}_{k}^{z}=\mathrm{DE}_{k}^{z} \cap \mathrm{BC}_{k}^{z} \subset \operatorname{Jet}_{k}^{z}$ of jets, satisfying both the equation (3.1.1) and the boundary conditions. For interior nodes we put $\mathrm{Sol}_{k}^{z}=$ $\mathrm{DE}_{k}^{z} \subset \mathrm{Jet}_{k}^{z}$, since there are no additional restrictions. For linear equations, $\mathrm{Sol}_{k}^{z}$ is an affine subspace of $\mathrm{Jet}_{k}^{z}$. We will assume that it can be parametrized by the free parameters $U_{\gamma}^{z}, \gamma \in \overline{\mathcal{B}}_{k}^{z} \subset \mathcal{B}_{k}^{z}$ at each node (recall that $U_{\gamma}^{z}$ corresponds to the derivative of order $\gamma$ at $z$ ). So we denote by $\overline{\mathcal{B}}_{k}^{z}$ the set of multi-indexes which form a coordinate system on $\mathrm{Sol}_{k}^{z}$.

Denote by $\widetilde{\mathrm{Sol}_{k}^{z}} z$ the linear space spanned by monomials from $\overline{\mathcal{B}}_{k}^{z}$. This is a linear space parallel to $\mathrm{Sol}_{k}^{z}$, but passing through the origin which cannot be true for $\mathrm{Sol}_{k}^{z}$ because of the boundary conditions described above. The whole jet satisfying the differential equation can be easily reconstructed by embedding when only free

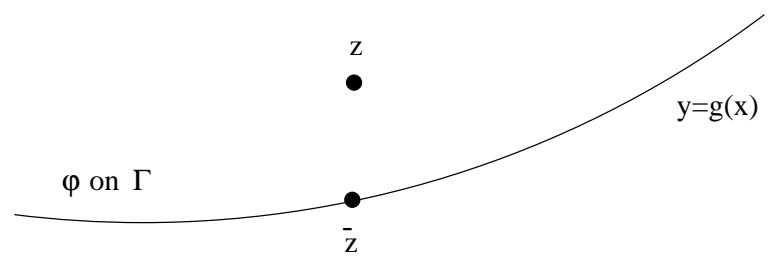

FIGURE 3.3.1 
components are given. We denote this operation by $E_{k}^{z}: \widetilde{\operatorname{Sol}_{k}^{z}} \rightarrow \operatorname{Jet}_{k}^{z}$. Its inverse is well defined on $\mathrm{Sol}_{k}^{z}$ and can be easily extended on the whole $\operatorname{Jet}_{k}^{z}$. Denote by $P_{k}^{z}$ this projection operator which gives only free components of any jet. This guarantees $P_{k}^{z} E_{k}^{z}$ to be the identity on $\widetilde{\operatorname{Sol}_{k}^{z}}$ and $E_{k}^{z} P_{k}^{z}$ to be the identity on $\operatorname{Sol}_{k}^{z}$.

3.4. Rough estimate. Consider the problem

$$
L u=0 \text { in } \Omega,\left.\quad u\right|_{\Gamma}=\varphi \text { on } \Gamma=\partial \Omega,
$$

where $L$ is a linear elliptic differential operator of second order. Denote the solution of (3.4.1) by $\bar{u}$ and assume that for any (smooth enough) $u$, the following inequality (an a priori estimate) is satisfied:

$$
\max _{\Omega}|u-\bar{u}| \leq c_{1}\left(\max _{\Omega}|L u|+\max _{\Gamma}|u-\varphi|\right) .
$$

From now on we fix an $h$-quasiregular grid $G=\left\{z_{1}, \ldots, z_{N}\right\}$ in $\Omega \subset \mathbb{R}^{n}$. Those $z_{j}$, for which the ball $B_{h}\left(z_{j}\right)$ (of radius $h$ centered at $z_{j}$ ) intersects $\Gamma$, we call boundary nodes of $G$.

At each interior point $z_{j} \in G$ we consider the subspace $\mathrm{Sol}_{k}^{z_{j}}=\mathrm{DE}_{k}^{z_{j}}$ of all the jets, satisfying the initial equation together with all its differential corollaries up to order $k$, parametrized by $U_{\gamma}^{z_{j}}, \gamma \in \overline{\mathcal{B}}_{k}^{z_{j}}$, as above. At each boundary point $z_{j}$ we consider the subspace $\mathrm{Sol}_{k}^{z_{j}}=\mathrm{DE}_{k}^{z_{j}} \cap \mathrm{BC}_{k}^{z_{j}}$ parametrized by $U_{\gamma}^{z_{j}}, \gamma \in \overline{\mathcal{B}}_{k}^{z_{j}}$. All these parameters $U_{\gamma}^{z_{j}}$ form the set of unknowns in our discretization.

For any choice of free parameters $U_{\gamma}^{z_{j}}$ we obtain a certain jet-function $U$ on $G$. As it was explained above, the solution procedure consists of minimization of $\mathrm{WF}(U)$ with respect to these free parameters. In this subsection we will not discuss the specific minimization procedures which can be used, but rather prove a general theorem, which gives a degree of approximation of a true solution $L$.

Denote by $c_{2}=\max _{x,|\beta| \leq k-2}\left|D^{\beta} L_{i}(x)\right|$ where $L_{i}$ are coefficients of the operator

Now we can formulate the following result which estimates a difference between true and formal solution in terms of the Whitney functional.

Theorem 3.4.1. Let $U$ be a jet-function from Sol at each gridnode. Assume that $\max _{\alpha, z_{j}}\left|U_{\alpha}^{z_{j}}\right| \leq c_{3}$ for each node $z_{j} \in G$ and $\operatorname{WF}(U) \leq M$. Then any function $v$, which is $c M h^{k+1}$ represented by $U$, satisfies for small $h$

$$
\max _{\Omega}|v-\bar{u}| \leq C\left(c M+c_{2} c_{3}\right) h^{k-1},
$$

where $C$ depends only on the dimension $n$ and order of jets $k$.

Proof. By the proof of the Whitney theorem there exists a smooth function $\tilde{v}$ with $k$-order jets coinciding at all gridnodes with the prescribed jet-function $U$. This means that equations (3.4.1) are satisfied up to order k-2: jet $_{k-2}^{z_{j}} L \tilde{v}=0$. Moreover the $k+1$-st derivative of $\tilde{v}$ does not exceed $c M$ in $\Omega$. This function is constructed as a sum of all the jets $U^{z}$ multiplied by partition of unity based on $h$-balls centered at gridnodes $G$.

Then $\left\|D^{k-1}(L \tilde{v})\right\| \leq C\left(c M+c_{2} c_{3}\right)$, since the highest derivatives (of order $k+1$ ) in this expression can be estimated by $M$ and all the derivatives of lower order arise when we differentiate several times the operator $L$ and the rest of $D^{k-1}$ acts on $\tilde{v}$ (by Leibnitz rule). 
Using Taylor decomposition of order $k-1$ of $L \tilde{v}$ at any gridpoint we obtain that all terms of order less than $k-1$ are zero. The residual term (taken in an intermediate point) is estimated above.

Since we assume that $U$ belongs to Sol also at boundary nodes, we have on $\Gamma$ $\max _{\Gamma}|\tilde{v}-\varphi| \leq c M h^{k+1}$. Together with the a priori estimate (3.4.2) this completes the proof, since any function $c M$-represented by $U$ can differ from $\tilde{v}$ by not more than $c M h^{k+1}$.

A similar result was obtained by Niijima [7].

The above result is too general to provide a good accuracy estimate. In what follows we demonstrate a special realization of the jet method which gives much higher precision for a special choice of the Whitney functional.

3.5. Multiscaling. The method of discretization we suggest typically has a very high order of accuracy. But the linear system of equations can be very stiff. Examples of condition numbers in case of the Dirichlet problem are given in Table 4.

We suggest a multiscaling procedure which allows one to overcome this difficulty with a minor increase of computational efforts. The idea is as follows.

The final matrix is badly conditioned because of the weights appearing always together with derivatives in the Taylor formula. One can use this fact in order to perform an effective pivoting (preconditioning) in the space of jets. The scaling multiplies the $\alpha$-th component of a jet in the residual equation by the coefficient $h^{k+1-|\alpha|}$ (compare with the norm on the Jet introduced in subsection 2.3).

Thus for $k=2$ the corresponding scaling matrix is

$$
\left(\begin{array}{cccccc}
h^{2}, & 0, & 0, & 0, & 0, & 0 \\
0, & h, & 0, & 0, & 0, & 0 \\
0, & 0, & h, & 0, & 0, & 0 \\
0, & 0, & 0, & 1, & 0, & 0 \\
0, & 0, & 0, & 0, & 1, & 0 \\
0, & 0, & 0, & 0, & 0, & 1
\end{array}\right) .
$$

This leads to the following effect - the condition numbers decrease essentially but the right-hand side elements increase. The idea is to apply this scaling to the residual terms at level $l$ only when the current approximation is properly found in terms of jets of order $l-1$. This means we can apply this scaling only when 0 -order derivatives have an $h^{l+1}$ order of magnitude, 1-order components are of order $h^{l}$, etc. Then the scaling will not lead to a drastic increase of the right-hand side since it is already properly scaled.

The whole algorithm takes the following form. First we perform only 0-order discretization and get a 0 order approximation. Then we embed this solution in the space of jets of order 1, obtain a new discretized system (of much higher dimension), perform scaling of the correction to be found and solve the resulting system with higher accuracy (typically one or two orders of $h$ ). Each next step consists of embedding, scaling and solving the residual system with corresponding accuracy. An example of this algorithm is explained in subsection 5.1

The geometrical picture behind this construction is the following. The Whitney functional is a nonnegative quadratic form on $\mathrm{JEF}_{l}$ space. It has eigenvalues of very different orders of magnitude. But for a section which is close to true functions, the discrepancies are correctly scaled; i.e., errors in values are of order $h^{l+1}$, in first derivatives of order $h^{l}$ and so on. This allows one to change the coordinate 
system in a way which makes a "ball" in the Whitney metric close to a ball in the natural metric (restricted on the subspace $\mathrm{SOL}_{l}$ ). This explains the effects we have discovered in the experimental part of this work.

\section{Optimal parameters for the Laplace and Poisson equations}

In this section we describe a specific realization of the general approach introduced above for the Laplace equation $\Delta u=0$ and Poisson equation $\Delta u=f$. We consider a discretization on a quasi-regular grid with mesh size $h$.

1. We are looking for the relaxation matrices $W_{l}$ and affine terms $R_{k}^{z}$ (for the Poisson equation), which express the solution jet at a given node through the solution jets at the neighboring nodes with the maximal order of accuracy:

$$
\operatorname{jet}_{k}^{z} u-\sum_{z_{l} \in N(z)} W_{l} \cdot \operatorname{jet}_{k}^{z_{l}} u-R_{k}^{z}=0 .
$$

2. We substitute into this equation the symbolic coefficients of jet ${ }_{k}^{z} u$ and express the coefficients of jet ${ }_{k}^{z_{l}} u$ through them as well. Then we solve the resulting system with respect to the coefficients $W_{l}$ and $R_{k}^{z}$ up to the highest possible order.

3. As a result we obtain a relaxation scheme. Its fixed point corresponds to the minimum of the Whithney functional constructed as a sum over the grid of squares of all the discrepancies.

4. The result of implementation of this scheme is a jet-function with high degree of smoothness (small Whitney functional). Actually the degree of smoothness (i.e., the order of discrepancy between neighboring jets) turns out to be much higher than for an arbitrary function represented by its $k$-order jets. We complete the representation finding the derivatives of orders higher than $k$ at each gridpoint. This is done by applying to the neighboring jets roughly the same matrices as in the relaxation procedure.

Below we give a more detailed description of each step.

4.1. Optimal weights calculation. Fix a node $z$ of the grid. Assume that at each neighboring node a $k$-order jet of a function $u$ is given which satisfies the equation (3.4.1). We will show how to find matrices $W_{l}$ depending only on the geometry of the stencil and the equation, such that for any function $u$ satisfying the equation (3.4.1) the jet-norm of the difference

$$
\operatorname{jet}_{k}^{z} u-\sum_{z_{l} \in N(z)} W_{l} \cdot \text { jet }_{k}^{z_{l}} u
$$

is as small as possible (in terms of order of $h$ ). These matrices define a relaxation scheme where on each step a jet $U_{k}^{z}$ is replaced by $\sum_{z_{l} \in N(z)} W_{l} \cdot U_{k}^{z_{l}}$.

We look for the coefficients of each matrix $W_{l}$ in the form $W_{\alpha \beta}^{z_{l}}=h^{|\beta|-|\alpha|} w_{\alpha \beta}^{l}$. Consider a symbolic jet $U_{K}^{z}$ placed at $z$ of an order $K(k \ll K)$ satisfying (3.4.1). This means that coordinates with $\gamma \in \overline{\mathcal{B}}_{k}^{z}$ are arbitrary and all others can be reconstructed by embedding $U_{K}^{z}=E_{K}^{z} \tilde{U}_{K}^{z}$, here $\tilde{U}_{K}^{z} \in \widetilde{\operatorname{Sol}_{K}^{z}}$.

Using the Taylor expansion we can express the coefficients of jet ${ }_{k}^{z_{l}} u$ through the coefficients of $\operatorname{jet}_{K}^{z} u$. This operation does not have to preserve the property to be a formal solution, so we perform the following. First take a set of free coefficients $\tilde{U}_{K}^{z}$ and embed it to $\operatorname{Sol}_{k}^{z}$. Then shift this jet to a node $z_{l} \in N(z)$. This can be written in our notation as $S_{K}^{z, z_{l}} E_{K}^{z} \tilde{U}_{K}^{z}$, the linear operator $S_{K}^{z, z_{l}}: \operatorname{Jet}_{K}^{z} \rightarrow \operatorname{Jet}_{K}^{z_{l}}$ being the shift matrix. The result is a $K$-order jet at the point $z_{l}$. To obtain a local 
solution of order $k$ we project it again on $\widetilde{\mathrm{Sol}_{k}^{z_{l}}}$ and embed the result into $\mathrm{Sol}_{k}^{z_{l}}$ by $E_{k}^{z_{l}}$. This gives $E_{k}^{z_{l}} P_{k}^{z_{l}} C_{K k} S_{K}^{z, z_{l}} E_{K}^{z} \tilde{U}_{K}^{z}$, with $C_{K k}$ the standard projection of $\operatorname{Sol}_{K}$ on its subspace $\mathrm{Sol}_{k}$. Generally the embedding is not necessarily a linear operation, which depends on the equation at each node, but for the Laplace equation at internal nodes it is a linear operator. The projection $P$ is always a linear operation.

If the manifold $\mathrm{Sol}_{k}^{z_{l}}$ is a linear subspace of $\mathrm{Jet}_{k}^{z_{l}}$ (Laplace equation, internal nodes for example) then the product $E_{k}^{z_{l}} P_{k}^{z_{l}} C_{K k}$ is equal to $C_{K k}$ on $\mathrm{Sol}_{K}^{z}$-a projection of a $K$-order jet-solution on its $k$-order part.

In case of Laplace equation this allows us to estimate instead of (4.1.1) only its free components:

$$
\tilde{U}_{k}^{z}-\sum_{z_{l} \in N(z)} P_{k}^{z_{l}} W_{l} E_{k}^{z_{l}} P_{k}^{z_{l}} C_{K k} S_{K}^{z, z_{l}} E_{K}^{z} \tilde{U}_{K}^{z}
$$

which we want to have the highest possible order in $h$.

Scalar form. Rewrite this matrix equation in a scalar form with the following scaling. Denote the $(\alpha, \beta)$ entry of the matrix $P_{k}^{z_{l}}$ by $h^{|\beta|-|\alpha|} p_{\alpha \beta}^{l}$, and the $(\gamma, \delta)$ entry of the matrix $S_{K}^{z, z_{l}}$ by $h^{|\delta|-|\gamma|} s_{\gamma \delta}^{l}$.

The system of equations defining $W$ (for the original Laplace equation at an internal node) becomes

$$
U_{\alpha}^{z}=\sum_{z_{l} \in N(z)} \sum_{\beta \in \overline{\mathcal{B}}_{k}^{z_{l}}} \sum_{\gamma \in \mathcal{B}_{k}^{z l}} \sum_{\delta \in \mathcal{B}_{K}^{z}} h^{|\beta|-|\alpha|} p_{\alpha \beta}^{l} \cdot h^{|\gamma|-|\beta|} w_{\beta \gamma}^{l} \cdot h^{|\delta|-|\gamma|} s_{\gamma \delta}^{l} \cdot U_{\delta}^{z} .
$$

Since these equalities must hold for all $U_{\alpha}^{x}$ up to the highest possible value $|\alpha|$ we can exclude $U$ and get a system for $w$ with $\alpha \in \overline{\mathcal{B}}_{k}^{z}$ :

$$
\sum_{\beta \in \overline{\mathcal{B}}_{k}^{z_{l}}} \sum_{\gamma \in \mathcal{B}_{k}^{z}} \sum_{\delta \in \mathcal{B}_{K}^{z}} p_{\alpha \beta}^{l} w_{\beta \gamma}^{l} s_{\gamma \delta}^{l}= \begin{cases}0, & \text { for } \delta \neq \alpha \\ 1, & \text { for } \delta=\alpha .\end{cases}
$$

There exists a maximal $K$ for which these equations are solvable. Then the error in $U_{\alpha}$, which cannot be avoided by using only jets of order $k$ with the same stencil, is of order $h^{K-|\alpha|}$.

We have considered two model cases. First, when the optimal weights matrices are assumed to be triangular means we use only derivatives of order $l$ and higher to find the $l$-th order derivative. Second (full), we consider this case without this assumption. The results of our calculations are given in Section 6 for both Dirichlet and Poisson problems.

Optimal parameters for the Poisson equation. For the Poisson equation $\Delta u=f$ the $\mathrm{Sol}_{k}^{z}$ subspace is not a linear subspace of $\mathrm{Jet}_{k}^{z}$ at each internal node $z$, but an affine subspace, which can be represented as a sum of harmonic jets and a jet $F_{k}^{z}$ corresponding to the right-hand side. For $k=2$ it has the form $F_{2}^{z}=(0,0,0,0,0, f(z))^{T}$. Thus to reconstruct a jet by its free coefficients, we use embedding of $\widetilde{\operatorname{Sol}_{k}^{z}}$ to $\operatorname{Jet}_{k}^{z}$ and add the vector corresponding to the right-hand side $E_{k}^{z} U_{k}^{z}+F_{k}^{z}$.

This leads to the following expression:

$$
\tilde{U}_{k}^{z}-\sum_{z_{l} \in N(z)} P_{k}^{z_{l}} W_{l}\left(E_{k}^{z_{l}} P_{k}^{z_{l}} C_{K k} S_{K}^{z, z_{l}}\left(E_{K}^{z} \tilde{U}_{K}^{z}+F_{K}^{z}\right)+F_{k}^{z_{l}}\right)+\tilde{R}_{k}^{z}
$$

Again we look for the optimal parameters: matrices $W_{l}$ and vectors $\tilde{R}_{k}^{z}$ which minimize the discrepancy up to the highest possible order. 
The best choice corresponds to the same linear operators $W_{l}$ and the affine part $\tilde{R}_{k}^{z}=-\sum_{z_{l} \in N(z)} P_{k}^{z_{l}} W_{l}\left(E_{k}^{z_{l}} P_{k}^{z_{l}} C_{K k} S_{K}^{z, z_{l}} F_{K}^{z}+F_{k}^{z}\right)$, which guarantees the best accuracy.

The relaxation procedure takes the form

$$
E_{k}^{z} P_{k}^{z}\left(\sum_{z_{l} \in N(z)} W_{l} U_{k}^{z_{l}}+R_{k}^{z}\right)+F_{k}^{z} \rightarrow U_{k}^{z(\text { new })} .
$$

4.2. Completion operator. As was mentioned above, the relaxation procedure usually leads to a k-jet solution with the discrepancy between the neighboring jets of order much higher than $h^{k+1}$. This means that the stencil also contains some information about higher derivatives.

To extract this information we solve the problem of how to reconstruct derivatives of order higher than 2 of a harmonic function when its 2-order jets are given at 5 neighboring nodes. In a general situation instead of harmonic function one can deal with a function satisfying some differential equation and consider jets of order $k$ as given.

In order to reconstruct the high order derivatives, the same scheme is used as for optimal weights. Denote by $Q_{l}$ the unknown matrices of reconstruction. They act on jets of order $k$ at all neighboring nodes in $N(z)$ and the node $z$ itself. The result should be a jet of order $K$ at the node $z$. Consider the difference

$$
\operatorname{jet}_{K}^{z} u-\sum_{z_{l} \in N(z) \cup\{z\}} Q_{l} \cdot \operatorname{jet}_{k}^{z_{l}} u .
$$

The dimension of matrices $Q$ is $\operatorname{dim} \operatorname{Jet}_{K} \times \operatorname{dim} \operatorname{Jet}_{k}$. It leads to a system similar to (4.1.2). We have solved this system symbolically (on a computer) for both situations in study - triangilar and full weights. The results of this calculation were used in the numerical experiments we have performed. Examples of solution of (4.2.1) are given in Section 6.

4.3. Boundary conditions. One of the central problems in our approach is an appropriate setting of boundary conditions. Indeed, having all the derivatives up to order $k$ as free parameters at each gridpoint, it would be natural to prescribe the values of all these derivatives on the boundary. However, the Dirichlet data provide us only the values of the derivatives in the direction of the boundary.

We did not try at this stage to investigate this problem mathematically, which certainly presents serious difficulties, since the Zigmund-Calderon operator, completing the Dirichlet boundary data to a full jet, is not a differential operator, but a pseudodifferential one (which means it is not local). Instead we split the problem in two parts:

1. We investigated the boundary data in the form of full jets. The main purpose here was to study the discretization error and accuracy at interior points.

Notice that although in the usual setting this problem is ill-posed, its restriction on the subspace of harmonic functions in a neighborhood of $\Omega$ is well defined.

2. We considered the usual Dirichlet data (jets in the boundary directions only). In this case the unknowns are the derivatives at the interior gridpoints and the transversal (and mixed) derivatives at the boundary gridpoints. The optimal relaxation matrices are computed in the same way as above.

We conjecture that our system is well posed. 
To confirm this conjecture experimentally, we have compared solutions of the same problems for Dirichlet and full jet boundary data (when we assume that all derivatives of the true solution are given on the boundary).

Both the accuracy and the condition numbers of these two approaches turned out to be approximately the same.

The results of our symbolic computation are provided in Section 6 .

For the second order jets the optimal relaxation matrices lead to a cancellation of all terms of order less than 12 (for values). The errors in the jet-form are $\left\{\frac{h^{12}}{12 !} U_{84},-\frac{h^{10}}{8 ! 462} U_{92}, \frac{h^{10}}{8 ! 462} U_{83},-\frac{h^{8}}{8 !} U_{82},-\frac{h^{8}}{9 !} U_{91}, \frac{h^{8}}{8 !} U_{82}\right\}$.

\section{EXPERIMENTAL RESULTS}

5.1. Discretized system for a fixed level. A few series of numerical experiments based on the proposed discretization scheme were performed. The model problem which we have considered was the Dirichlet problem in the rectangle $[0,1] \times[0,1] \subset$ $\mathbb{R}^{2}$. Of course for this concrete problem there are known effective methods, but we actually do not use the special geometry of the domain, so it is hoped that the same results are true for more complicated domains.

We have considered cases $k=0,1,2$. The case $k=0$ corresponds to the standard discretization - 5-points Laplacian. As a true solution we choose the following two harmonic functions: $u_{7}(x, y)=x^{7}-21 x^{5} y^{2}+35 x^{3} y^{4}-7 x y^{6}$ and $u_{-3}(x, y)=$ $e^{3 x} \sin (3 y)$. We use their values and derivatives as given boundary conditions.

Consider an $N \times N$ regular grid $G$ with stepsize $h=\frac{1}{N-1}$, and $4(N-1)$ nodes exactly on the boundary.

We use two types of boundary conditions. First, we use full jets when at each boundary node the whole jet of an unknown function is given. Second, we use boundary jets when at each boundary point only derivatives along the boundary are given. This case corresponds to a real Dirichlet problem, and the first one is used for study of spectral and approximative properties of the discretization scheme we suggest.

Let $\bar{U} \in \mathrm{JEF}_{k}$ be an exact solution of the discretized problem. Denote by $P$ projection of a global jet-function on the subspace $\widetilde{\mathrm{SOL}}$ which is a parallel translation of the affine subspace SOL to the origin (it is spanned by monomials from $\left.\overline{\mathcal{B}}_{k}^{z}\right)$. This can be done easily since we have fixed in each subspace $\mathrm{Jet}_{k}^{z}$ a set of free coefficients $\overline{\mathcal{B}}_{k}^{z}$ which form a coordinate system on $\mathrm{Sol}_{k}^{z}$. Thus, the action of $P$ is defined at each node.

The subspace SOL is an affine subspace in JEF. Fix a vector $V$ in it, such that $P V=0$, and a linear operator $E$ which reconstruct jet-function by its free components: $V+E X \in \mathrm{SOL}, P E=i d_{\widetilde{\mathrm{SOL}}}$.

The optimal weights found before define a linear operator on JEF which we denote by $A$. It has a block-bandwise structure, and the jet-function $\bar{U}$ we are looking for is its fixed point

$$
A \bar{U}=\bar{U}
$$

Moreover, the vector $\bar{U}$ should satisfy the boundary and differential relations; therefore we have to find this vector in the form $V+E X$. Here $V$ represents an affine part of boundary data and $X$ is an arbitrary vector of free coefficients (i.e., from 
$\widetilde{\mathrm{SOL}})$. Then (5.1.1) takes the form

$$
A(V+E X)=V+E X
$$

or

$$
P A V+P A E X=P V+P E X,
$$

which is equivalent to

$$
(I-P A E) X=P A V
$$

This is the final equation for $X$; its solution gives the exact solution of the discretized problem $V+E X$.

The difficulty of solving this linear system numerically depends on the spectral properties of the operator $I-P A E$ and the order of magnitude of $P A V$ when $h$ tends to 0 . Since the inverse matrix should be applied to the vector $P A V$ we have also measured its maximal elements which could influence the final accuracy of any iterative algorithm. These results are represented in the tables below and in Section 6.

5.2. Poisson equation. Another series of experiments were performed for the Poisson problem in the same domain. The equation $\Delta u=f$ was considered. The right-hand side and boundary values were chosen in such a way that the true analytic solution was $u(x, y)=x^{7}-21 x^{5} y^{2}+35 x^{3} y^{4}-7 x y^{6}+\sin (4 x y)$ in the first case and $u(x, y)=e^{3 x} \sin (3 y)+\sin (4 x y)$ in the second. Again several grids were considered with the same sizes: $N=3,5,9,17$ which corresponds to $h=\frac{1}{2}, \frac{1}{4}, \frac{1}{8}, \frac{1}{16}$.

As was shown in (4.1.5), the optimal weights found for the Laplace equation are valid also in this case, but the final equation for the unknown jet-function also involves the right-hand side.

Let $\bar{U}$ be the true solution of the discretized problem. Denote by $X \in \widetilde{\mathrm{SOL}}$ the vector of free coefficients to be found, and the affine components are $F+V$, where $F$ arises from the right-hand side of the equation (see $F_{k}^{z}$ in (4.1.3)) and $V$ appears from boundary data (as in the Dirichlet case). The embedding formula takes the following form: $U=F+E X \in \mathrm{SOL}$ and the equation for optimal smoothing also involves an affine component (instead of (5.1.1))

$$
A \bar{U}+R=\bar{U},
$$

which can be rewritten as

$$
A(F+V)+A E X+R=F+V+E X .
$$

Project the equation on the subspase of free coefficients

$$
P A(F+V)+P A E X+P R=P F+P V+P E X,
$$

which is equivalent to

$$
X-P A E X=P R+P A F+P A V-P F
$$

(recall that $P E=i d_{\widetilde{\mathrm{SOL}}}$ and $P V=0$ ). Finally,

$$
X=(I-P A E)^{-1} \cdot(P R+P(A-I) F+P A V) .
$$

The results of our experiments are represented in the tables below and in Section 6. They include discretization errors (difference between true solution of the discretized problem and the analytic one at nodes), condition numbers of the matrix 
$(I-P A E)$ (which we have to invert), and the biggest values of the right-hand side, i.e., of the vector $(P R+P(A-I) F+P A V)$.

5.3. Multiscale implementation. The singular values of the matrix $I-P A E$ have very different orders of magnitude. Some of them, which correspond to higher derivatives, are of order $h^{0}$, but some, which correspond to lower derivatives, are of order $h^{k}$. The multiscale method we suggest involves $k+1$ steps. At each step the problem is solved at a current level $l$, then we perform scaling and increase the level by one (from $l$ to $l+1$ ).

Denote the 0 -level discretization system by $C_{0} X_{0}=B_{0}$. We solve it up to accuracy $h^{0}$. Next we embed the vector $X_{0} \in \mathrm{JEF}_{0}^{G}$ into $\mathrm{JEF}_{1}^{G}$. Write this operation as $G_{0}\left(E_{0} X_{0}+V_{0}\right)$, where $G_{0}$ is the embedding matrix, $E_{0}$ maps $\widetilde{\mathrm{SOL}_{0}^{G}}$ to $\mathrm{JEF}_{0}^{G}$ and $V_{0}$ is the boundary data (affine component).

Denote the new unknown vector by $Y_{1}$ and scale it. The new system takes the form $C_{1} X_{1}=B_{1}$, where $X_{1}=G_{0}\left(E_{0} X_{0}+V_{0}\right)+D_{1} Y_{1}$. So the new matrix is $C_{1} D_{1}$ and the new right-hand side is $B_{1}-C_{1} G_{0}\left(E_{0} X_{0}+V_{0}\right)$.

In an analogous way we solve this system approximately and increase the order of jets by one. Again, from $C_{2} X_{2}=B_{2}$ we arrive at $C_{2} D_{2} Y_{2}=B_{2}-C_{2} G_{1}\left(E_{1} X_{1}+V_{1}\right)$. The condition numbers of the new operators $C_{2} D_{2}, C_{1} D_{1}, C_{0}$ and maximal values of the new right-hand sides are given in Tables 1-9 and in Section 6.

The embedding operators can be constructed in different ways. We have used in our computational scheme the following embeddings $\mathrm{JEF}_{i} \rightarrow \mathrm{JEF}_{i+1}$ from level 0 level 1:

$$
\begin{aligned}
& \bar{U}_{00}^{z_{5}}=U_{00}^{z_{5}}, \\
& \bar{U}_{10}^{z_{5}}=\left(U_{00}^{z_{4}}-U_{00}^{z_{2}}\right) / 2, \\
& \bar{U}_{01}^{z_{5}}=\left(U_{00}^{z_{1}}-U_{00}^{z_{3}}\right) / 2 ;
\end{aligned}
$$

and embedding from level 1 to level 2:

$$
\begin{aligned}
& \bar{U}_{00}^{z_{5}}=U_{00}^{z_{5}}, \\
& \bar{U}_{10}^{z_{5}}=U_{10}^{z_{5}}, \\
& \bar{U}_{01}^{z_{5}}=U_{01}^{z_{5}}, \\
& \bar{U}_{20}^{z_{5}}=-\frac{1}{h^{2}}\left(U_{00}^{z_{1}}+U_{00}^{z_{3}}-U_{00}^{z_{4}}-U_{00}^{z_{2}}\right)-\frac{1}{4 h}\left(U_{01}^{z_{3}}-U_{01}^{z_{1}}+U_{10}^{z_{4}}-U_{10}^{z_{2}}\right), \\
& \bar{U}_{11}^{z_{5}}=\frac{1}{4 h}\left(U_{10}^{z_{1}}-U_{10}^{z_{3}}+U_{01}^{z_{4}}-U_{01}^{z_{2}}\right), \\
& \bar{U}_{02}^{z_{5}}=\frac{1}{h^{2}}\left(U_{00}^{z_{1}}+U_{00}^{z_{3}}-U_{00}^{z_{4}}-U_{00}^{z_{2}}\right)+\frac{1}{4 h}\left(U_{01}^{z_{3}}-U_{01}^{z_{1}}+U_{10}^{z_{4}}-U_{10}^{z_{2}}\right) .
\end{aligned}
$$

5.4. Explanation of tables. All calculations were performed with the Mathematica 2.2 program on an IBM RS6000 computer. For some values of parameters the resulting linear system was too large (more than $1000 \times 1000)$ and the program did not succeed in inverting such a matrix. This is marked by $\mathrm{a} \star$ in the corresponding position of each table. 
Discretization errors, Dirichlet problem. In Tables 1-3 we give the results of the comparison of the solution of the discretized problem and the analytic one. The following solution was used: $u(x, y)=e^{3 x} \sin 3 y$. The domain $\Omega=[0,1] \times[0,1]$. This function and its first and second derivatives have the range $[0,20],[-60,60]$, $[-180,180]$.

Table 4 represents the condition numbers of the linear systems we have to solve for the full method and for its multiscale implementation.

Tables 5-9 represent the convergence rate obtained. The rest of our experimental results can be found in Section 6.

One can find similar experimental results for the Poisson equation as well as more detailed error analysis (maximum norm error, etc.) in Section 6.

As far as the observed rates of convergence are concerned, let us compute (following the referee suggestion) the following quantity.

TABle 1. Dirichlet problem. Discretization errors in jet-form. Analytic solution: $e^{3 x} \sin 3 y$. Triangular method, boundary jets fixed.

\begin{tabular}{|c|c|c|c|}
\hline $\mathrm{N} \backslash \mathrm{k}$ & 0 & 1 & 2 \\
\hline 3 & $\{0.95\}$ & $\left\{2.810^{-3}, 2.8,0.2\right\}$ & $\left\{1.10^{-6}, 8.510^{-3}, 6.10^{-4}, 8.5,0.6\right\}$ \\
\hline 5 & $\{0.3\}$ & $\{0.03,0.89,0.36\}$ & $\left\{2.10^{-3}, 0.09,0.07,2.67,1.\right\}$ \\
\hline 9 & $\{0.08\}$ & $\{0.0079,0.23,0.096\}$ & $\left\{7.710^{-4}, 0.02,0.02,0.7,0.3\right\}$ \\
\hline 17 & $\{0.02\}$ & $\{0.002,0.06,0.02\}$ & $\star$ \\
\hline
\end{tabular}

TABle 2. Dirichlet problem. Discretization errors in jet-form. Analytic solution: $e^{3 x} \sin 3 y$. Full method, boundary jets fixed.

\begin{tabular}{|c|c|c|c|}
\hline$N \backslash \mathrm{k}$ & 0 & 1 & 2 \\
\hline 3 & $\{0.95\}$ & $\left\{2.810^{-3}, 5.110^{-2}, 3.510^{-3}\right\}$ & $\left\{1.210^{-6}, 4.210^{-5}, 2.910^{-6}, 0.026,2.10^{-4}\right\}$ \\
\hline 5 & $\{0.3\}$ & $\left\{7.10^{-5}, 1.910^{-3}, 1.310^{-3}\right\}$ & $\left\{1.210^{-6}, 3.310^{-6}, 7.710^{-7}, 9.10^{-4}, 2.210^{-5}\right\}$ \\
\hline 9 & $\{0.08\}$ & $\left\{2.110^{-6}, 4.610^{-5}, 3.410^{-5}\right\}$ & $\left\{6.10^{-10}, 2.310^{-9}, 4.910^{-9}, 1.610^{-6}, 1.10^{-7}\right\}$ \\
\hline 17 & $\{0.02\}$ & $\left\{4.010^{-8}, 9.010^{-7}, 6.410^{-7}\right\}$ & $\star$ \\
\hline
\end{tabular}

TABle 3. Dirichlet problem. Discretization errors in jet-form. Analytic solution: $e^{3 x} \sin 3 y$. Full method, true Dirichlet boundary data.

\begin{tabular}{|c|c|c|c|}
\hline$N \backslash k$ & 0 & 1 & 2 \\
\hline 3 & $\{0.95\}$ & $\left\{2.10^{-3}, 6.710^{-3}, 0.05\right\}$ & $\left\{3.310^{-4}, 0.003,0.001,0.06,0.02\right\}$ \\
\hline 5 & $\{0.3\}$ & $\left\{1.510^{-4}, 5.510^{-3}, 1.10^{-3}\right\}$ & $\left\{3.210^{-7}, 3.110^{-6}, 1.210^{-6}, 2.310^{-4}, 1.210^{-4}\right\}$ \\
\hline 9 & $\{0.08\}$ & $\left\{5.110^{-6}, 2.10^{-4}, 3.810^{-5}\right\}$ & $\left\{4.110^{-10}, 1.310^{-8}, 3.210^{-9}, 1.610^{-6}, 1.310^{-6}\right\}$ \\
\hline 17 & $\{0.02\}$ & $\left\{1.110^{-7}, 9.110^{-6}, 1.10^{-6}\right\}$ & $\star$ \\
\hline
\end{tabular}


TABLE 4. Condition numbers, full method, the whole boundary jets are fixed.

\begin{tabular}{|c|c|c|c|c|c|c|c|}
\hline \multicolumn{4}{c}{ without multiscaling } & \multicolumn{3}{c|}{ with multiscaling } \\
\hline $\mathrm{N} \backslash \mathrm{k}$ & 0 & 1 & 2 & & 0 & 1 & 2 \\
\hline 3 & 1 & 1 & 1 & & 1 & 1 & 1 \\
\hline 5 & 5.83 & 105.5 & $3.710^{6}$ & & 5.83 & 14.7 & $1.5610^{4}$ \\
\hline 9 & 25.27 & 1341. & $\star$ & & 25.27 & 57.5 & $5.5610^{4}$ \\
\hline 17 & 103.1 & $1.2310^{4}$ & $\star$ & & 103.1 & 230 & $\star$ \\
\hline
\end{tabular}

TABLE 5. Dirichlet problem triangular method.

\begin{tabular}{|l|c|c|c|}
\hline $\mathrm{h} \backslash \mathrm{k}$ & 0 & 1 & 2 \\
\hline$\frac{1}{2}: \frac{1}{4}$ & 1.66 & -3.4 & -11 \\
\hline$\frac{1}{4}: \frac{1}{8}$ & 1.91 & 1.93 & 1.4 \\
\hline$\frac{1}{8}: \frac{1}{16}$ & 2 & 2.0 & $\star$ \\
\hline
\end{tabular}

TABLE 6. Dirichlet problem full method.

\begin{tabular}{|l|c|c|c|}
\hline $\mathrm{h} \backslash \mathrm{k}$ & 0 & 1 & 2 \\
\hline$\frac{1}{2}: \frac{1}{4}$ & 1.7 & 5.4 & 0 \\
\hline$\frac{1}{4}: \frac{1}{8}$ & 1.9 & 5.1 & 11 \\
\hline$\frac{1}{8}: \frac{1}{16}$ & 2 & 5.7 & $\star$ \\
\hline
\end{tabular}

TABle 7. Poisson problem triangular method.

\begin{tabular}{|l|c|c|c|}
\hline $\mathrm{h} \backslash \mathrm{k}$ & 0 & 1 & 2 \\
\hline$\frac{1}{2}: \frac{1}{4}$ & 1.7 & -3 & -10 \\
\hline$\frac{1}{4}: \frac{1}{8}$ & 1.9 & 1.9 & 1.2 \\
\hline$\frac{1}{8}: \frac{1}{16}$ & 2 & 2 & $\star$ \\
\hline
\end{tabular}

Each of our tables contains the jet discretization errors for the same problem with the gridstep $h$ equal to $\frac{1}{2}, \frac{1}{4}, \frac{1}{8}, \frac{1}{16}$, respectively. Compute for each gridstep $h$ and each $k=0,1,2$, the convergence rate

$$
\frac{1}{\ln 2} \ln \left(\frac{\operatorname{error}(h)}{\operatorname{error}\left(\frac{h}{2}\right)}\right) \text {. }
$$

We get Tables 5-9 (only the errors in zero-order term are used). 
TABLE 8. Poisson problem full method.

\begin{tabular}{|l|c|c|c|}
\hline $\mathrm{h} \backslash \mathrm{k}$ & 0 & 1 & 2 \\
\hline$\frac{1}{2}: \frac{1}{4}$ & 1.7 & 5.1 & -1.1 \\
\hline$\frac{1}{4}: \frac{1}{8}$ & 1.9 & 5.1 & 8.8 \\
\hline$\frac{1}{8}: \frac{1}{16}$ & 2 & 5.7 & $\star$ \\
\hline
\end{tabular}

TABLE 9. Dirichlet problem full method, true boundary data.

\begin{tabular}{|l|c|c|c|}
\hline $\mathrm{h} \backslash \mathrm{k}$ & 0 & 1 & 2 \\
\hline$\frac{1}{2}: \frac{1}{4}$ & 1.7 & 3.7 & 10 \\
\hline$\frac{1}{4}: \frac{1}{8}$ & 1.9 & 4.9 & 9.6 \\
\hline$\frac{1}{8}: \frac{1}{16}$ & 2 & 5.5 & $\star$ \\
\hline
\end{tabular}

We agree with the referee's observation that these results (also seemingly the case $k=2, h=\frac{1}{16}$ has not settled down in the asymptotic range yet) suggest that the triangular method restricts the rate of convergence to the second order.

On the other hand, the above results support the high convergence rate for the full method, both for the full-jet boundary data and for the "true Dirichlet data". (A possible explanation of such a big difference between the triangular and the full methods may be that the relaxation scheme local error for the full scheme is of order $\left(h^{12}, h^{10}, h^{8}\right)$ for the values, the first and the second derivatives, respectively, while for the triangular scheme this error is of order $\left(h^{12}, h^{8}, h^{4}\right)$. See Section 6 for results of symbolic calculations.

\section{Supplement}

To find the optimal parameters $W_{l}$ and $R^{z}$ described in Section 4, we have solved the corresponding equations symbolically. This section contains results in a final form of the optimal weights for different nodes and schemes. A regular grid with stepsize $h$ was used.

6.1. Internal nodes. Here we list the matrices $W_{l}$ defined above which we have obtained by symbolic calculations. We assume that the grid $G$ is regular with stepsize $h$ and for each internal node its 4 neighbors are defined by the standard stencil. The neighbors are ennumerated in the following way: 1-North, 2-East, 3-South, 4-West, 5-central node.

When $\underline{k=0}$, obviously the standard weights $W_{1}=W_{2}=W_{3}=W_{4}=\frac{1}{4}$ for both full and triangular cases are best possible. They lead to errors in values of order $\frac{h^{2}}{4 !} U_{40}$.

Triangular case $k=1$. Optimal weights (matrices $W$ ) are

$$
\left\{\left(\begin{array}{ccc}
\frac{1}{4}, & 0, & \frac{-h}{16} \\
0, & \frac{1}{4}, & 0 \\
0, & 0, & \frac{1}{4}
\end{array}\right),\left(\begin{array}{ccc}
\frac{1}{4}, & \frac{h}{16}, & 0 \\
0, & \frac{1}{4}, & 0 \\
0, & 0, & \frac{1}{4}
\end{array}\right),\left(\begin{array}{ccc}
\frac{1}{4}, & 0, & \frac{h}{16} \\
0, & \frac{1}{4}, & 0 \\
0, & 0, & \frac{1}{4}
\end{array}\right),\left(\begin{array}{ccc}
\frac{1}{4}, & \frac{-h}{16}, & 0 \\
0, & \frac{1}{4}, & 0 \\
0, & 0, & \frac{1}{4}
\end{array}\right)\right\} .
$$




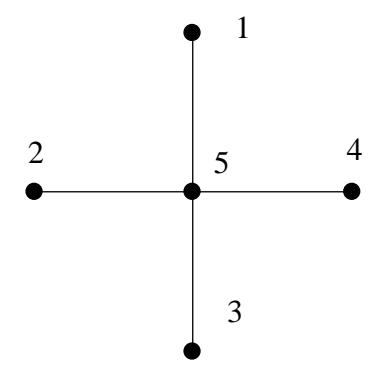

FiguRE 6.1 .1

Corresponding errors (in jet-form): $\left\{-\frac{h^{8}}{8 !} U_{80}, \frac{h^{4}}{4 !} U_{50}, \frac{h^{4}}{4 !} U_{41}\right\}$.

Full case $k=1$. Optimal weights (matrices $W$ ) are

$$
\left\{\left(\begin{array}{ccc}
\frac{1}{4}, & 0, & \frac{-h}{16} \\
0, & \frac{1}{24}, & 0 \\
\frac{5}{8 h}, & 0, & \frac{-1}{6}
\end{array}\right),\left(\begin{array}{ccc}
\frac{1}{4}, & \frac{h}{16}, & 0 \\
\frac{-5}{8 h}, & \frac{-1}{6}, & 0 \\
0, & 0, & \frac{1}{24}
\end{array}\right),\left(\begin{array}{ccc}
\frac{1}{4}, & 0, & \frac{h}{16} \\
0, & \frac{1}{24}, & 0 \\
\frac{-5}{8 h}, & 0, & \frac{-1}{6}
\end{array}\right),\left(\begin{array}{ccc}
\frac{1}{4}, & \frac{-h}{16}, & 0 \\
\frac{5}{8 h}, & \frac{-1}{6}, & 0 \\
0, & 0, & \frac{1}{24}
\end{array}\right)\right\} .
$$

Corresponding errors (in jet-form): $\left\{-\frac{h^{8}}{8 !} U_{80}, \frac{h^{6}}{3024} U_{70}, \frac{h^{6}}{3024} U_{61}\right\}$.

Triangular case $k=2$. Optimal weights are

$$
\begin{gathered}
W_{1}=\left(\begin{array}{cccccc}
\frac{1}{4}, & 0, & -t_{2}, & c_{1}-t_{3}, & 0, & c_{1}+t_{3} \\
0, & \frac{1}{4}, & 0, & 0, & \frac{-h}{16}, & 0 \\
0, & 0, & \frac{1}{4}, & \frac{h}{32}, & 0, & \frac{-h}{32} \\
0, & 0, & 0, & \frac{1}{4}, & 0, & 0 \\
0, & 0, & 0, & 0, & \frac{1}{4}, & 0 \\
0, & 0, & 0, & 0, & 0, & \frac{1}{4}
\end{array}\right), \\
W_{2}=\left(\begin{array}{cccccc}
\frac{1}{4}, & t_{2}, & 0, & c_{2}+t_{3}, & 0, & c_{2}-t_{3} \\
0, & \frac{1}{4}, & 0, & \frac{h}{32}, & 0, & \frac{-h}{32} \\
0, & 0, & \frac{1}{4}, & 0, & \frac{h}{16}, & 0 \\
0, & 0, & 0, & \frac{1}{4}, & 0, & 0 \\
0, & 0, & 0, & 0, & \frac{1}{4}, & 0 \\
0, & 0, & 0, & 0, & 0, & \frac{1}{4}
\end{array}\right), \\
W_{3}= \\
W_{4}=\left(\begin{array}{cccccc}
\frac{1}{4}, & 0, & t_{2}, & c_{1}-t_{3}, & 0, & c_{1}+t_{3} \\
0, & \frac{1}{4}, & 0, & 0, & \frac{h}{16}, & 0 \\
0, & 0, & \frac{1}{4}, & \frac{-h}{32}, & 0, & \frac{h}{32} \\
0, & 0, & 0, & \frac{1}{4}, & 0, & 0 \\
0, & 0, & 0, & 0, & \frac{1}{4}, & 0 \\
0, & 0, & 0, & 0, & 0, & \frac{1}{4}
\end{array}\right), \\
\left.\begin{array}{cccccc}
\frac{1}{4}, & t_{2}, & 0, & c_{2}+t_{3}, & 0, & c_{2}-t_{3} \\
0, & \frac{1}{4}, & 0, & \frac{-h}{32}, & 0, & \frac{h}{32} \\
0, & 0, & \frac{1}{4}, & 0, & \frac{-h}{16}, & 0 \\
0, & 0, & 0, & \frac{1}{4}, & 0, & 0 \\
0, & 0, & 0, & 0, & \frac{1}{4}, & 0 \\
0, & 0, & 0, & 0, & 0, & \frac{1}{4}
\end{array}\right),
\end{gathered}
$$


where $c_{1}, c_{2}$ are arbitrary constants which we choose to be 0 , and $t_{2}=11 \mathrm{~h} / 128$, $t_{3}=h^{2} / 256$. Corresponding errors (in jet-form):

$$
\left\{\frac{h^{12}}{12 !} U_{84},-\frac{h^{8}}{8 !} U_{90},-\frac{h^{8}}{8 !} U_{81}, \frac{h^{4}}{4 !} U_{60} \frac{h^{4}}{4 !} U_{51},-\frac{4}{4 !} U_{60}\right\} .
$$

Full case $k=2$. Optimal weights are

$$
\begin{aligned}
W_{1} & =\left(\begin{array}{cccccc}
\frac{1}{4}, & 0, & -t_{2}, & c_{1}-t_{3}, & 0, & c_{1}+t_{3} \\
0, & t_{7}, & 0, & 0, & -t_{8}, & 0 \\
t_{0}, & 0, & -t_{9}, & 0, & 0, & t_{10} \\
-t_{4}, & 0, & -t_{5}, & t_{6}, & 0, & 0 \\
0, & t_{11}, & 0, & 0, & \frac{-1}{16}, & 0 \\
t_{4}, & 0, & t_{5}, & 0, & 0, & t_{6}
\end{array}\right), \\
W_{2} & =\left(\begin{array}{cccccc}
\frac{1}{4}, & t_{2}, & 0, & c_{2}+t_{3}, & 0, & c_{2}-t_{3} \\
-t_{0}, & -t_{9}, & 0, & -t_{10}, & 0, & 0 \\
0, & 0, & t_{7}, & 0, & t_{8}, & 0 \\
t_{4}, & -t_{5}, & 0, & t_{6}, & 0, & 0 \\
0, & 0, & -t_{11}, & 0, & \frac{-1}{16}, & 0 \\
-t_{4}, & t_{5}, & 0, & 0, & 0, & t_{6}
\end{array}\right), \\
W_{3} & =\left(\begin{array}{cccccc}
\frac{1}{4}, & 0, & t_{2}, & c_{1}-t_{3}, & 0, & c_{1}+t_{3} \\
0, & t_{7}, & 0, & 0, & t_{8}, & 0 \\
-t_{0}, & 0, & -t_{9}, & 0, & 0, & -t_{10} \\
-t_{4}, & 0, & t_{5}, & t_{6}, & 0, & 0 \\
0, & -t_{11}, & 0, & 0, & \frac{-1}{16}, & 0 \\
t_{4}, & 0, & -t_{5}, & 0, & 0, & t_{6}
\end{array}\right), \\
W_{4}= & \left(\begin{array}{cccccc}
\frac{1}{4}, & -t_{2}, & 0, & c_{2}+t_{3}, & 0, & c_{2}-t_{3} \\
t_{0}, & -t_{9}, & 0, & t_{10}, & 0, & 0 \\
0, & 0, & t_{7}, & 0, & -t_{8}, & 0 \\
t_{4}, & t_{5}, & 0, & t_{6}, & 0, & 0 \\
0, & 0, & t_{11}, & 0, & \frac{-1}{16}, & 0 \\
-t_{4}, & -t_{5}, & 0, & 0, & 0, & t_{6}
\end{array}\right),
\end{aligned}
$$

where $c_{1}, c_{2}$ are arbitrary constants which we choose to be 0 , and $t_{2}=11 h / 128, t_{3}=$ $h^{2} / 256, t_{4}=75 / 8 h^{2}, t_{5}=-165 / 32 h, t_{6}=23 / 32, t_{7}=11 / 224, t_{8}=h / 112, t_{9}=$ $113 / 448, t_{10}=11 h / 448, t_{11}=5 / 16 h$.

The corresponding errors (in jet-form):

$$
\left\{\frac{h^{12}}{12 !} U_{84},-\frac{h^{10}}{8 ! 462} U_{92}, \frac{h^{10}}{8 ! 462} U_{83},-\frac{h^{8}}{8 !} U_{82},-\frac{h^{8}}{9 !} U_{91}, \frac{h^{8}}{8 !} U_{82}\right\}
$$

We have given all the optimal weights for internal nodes of a regular grid (four neighbors).

6.2. Boundary optimal weights. For boundary nodes the matrices $W_{l}$ have another form, since there are only 3 neighbors, which we ennumerate in the following way.

The last matrix in each case corresponds to 5 -th neighbor (that is, to the node itself). They correspond to the derivatives along boundaries which are given as boundary data. 


\section{Numeration of neighbors at boundary nodes}

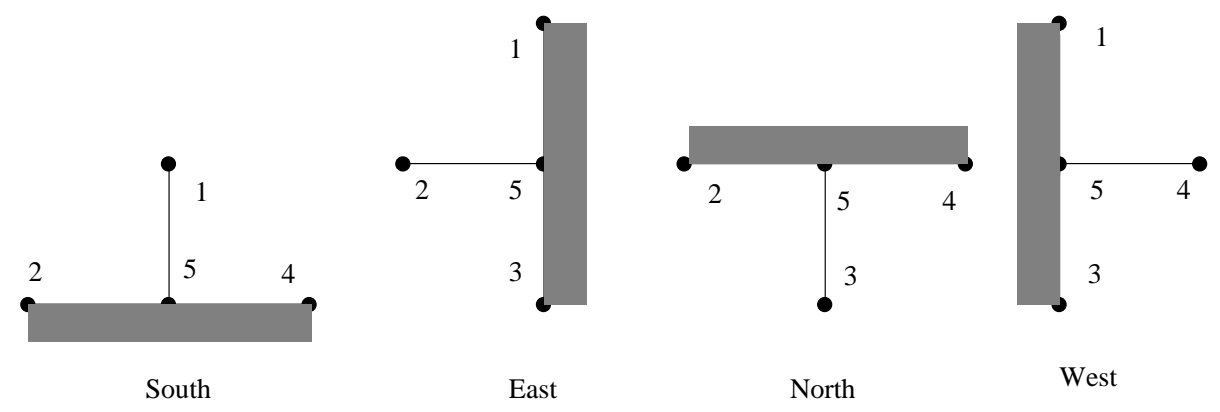

FiguRE 6.2.1

Triangular case $k=1$ boundary nodes. Optimal weights (matrices $W$ ) are:

South boundary (neighbors 1,2,4,5):

$$
\left\{\left(\begin{array}{ccc}
0, & 0, & 0 \\
0, & 0, & 0 \\
0, & 0, & \frac{1}{2}
\end{array}\right),\left(\begin{array}{ccc}
0, & 0, & 0 \\
0, & 0, & 0 \\
0, & \frac{-1}{4}, & \frac{1}{4}
\end{array}\right),\left(\begin{array}{ccc}
0, & 0, & 0 \\
0, & 0, & 0 \\
0, & \frac{1}{4}, & \frac{1}{4}
\end{array}\right),\left(\begin{array}{ccc}
1, & 0, & 0 \\
0, & 1, & 0 \\
0, & 0, & 0
\end{array}\right)\right\} ;
$$

North boundary (neighbors $2,3,4,5$ ):

$$
\left\{\left(\begin{array}{ccc}
0, & 0, & 0 \\
0, & 0, & 0 \\
0, & \frac{1}{4}, & \frac{1}{4}
\end{array}\right),\left(\begin{array}{ccc}
0, & 0, & 0 \\
0, & 0, & 0 \\
0, & 0, & \frac{1}{2}
\end{array}\right),\left(\begin{array}{ccc}
0, & 0, & 0 \\
0, & 0, & 0 \\
0, & \frac{-1}{4}, & \frac{1}{4}
\end{array}\right),\left(\begin{array}{ccc}
1, & 0, & 0 \\
0, & 1, & 0 \\
0, & 0, & 0
\end{array}\right)\right\} ;
$$

East boundary (neighbors $1,2,3,5$ ):

$$
\left\{\left(\begin{array}{ccc}
0, & 0, & 0 \\
0, & \frac{1}{4}, & \frac{-1}{4} \\
0, & 0, & 0
\end{array}\right),\left(\begin{array}{lll}
0, & 0, & 0 \\
0, & \frac{1}{2}, & 0 \\
0, & 0, & 0
\end{array}\right),\left(\begin{array}{ccc}
0, & 0, & 0 \\
0, & \frac{1}{4}, & \frac{1}{4} \\
0, & 0, & 0
\end{array}\right),\left(\begin{array}{lll}
1, & 0, & 0 \\
0, & 0, & 0 \\
0, & 0, & 1
\end{array}\right)\right\} ;
$$

West boundary (neighbors 1,3,4,5):

$$
\left\{\left(\begin{array}{ccc}
0, & 0, & 0 \\
0, & \frac{1}{4}, & \frac{1}{4} \\
0, & 0, & 0
\end{array}\right),\left(\begin{array}{ccc}
0, & 0, & 0 \\
0, & \frac{1}{4}, & \frac{-1}{4} \\
0, & 0, & 0
\end{array}\right),\left(\begin{array}{ccc}
0, & 0, & 0 \\
0, & \frac{1}{2}, & 0 \\
0, & 0, & 0
\end{array}\right),\left(\begin{array}{ccc}
1, & 0, & 0 \\
0, & 0, & 0 \\
0, & 0, & 1
\end{array}\right)\right\} .
$$

Corresponding errors in components of first-order derivatives are of order $\frac{h^{3}}{6} U_{40}, \frac{h^{3}}{6} U_{31}$.

Full case $k=1$ boundary nodes. Optimal weights (matrices $W$ ) are:

South boundary (neighbors $1,2,4,5)$ :

$$
\left\{\left(\begin{array}{ccc}
0, & 0, & 0 \\
0, & 0, & 0 \\
\frac{5}{4 h}, & 0, & \frac{-1}{3}
\end{array}\right),\left(\begin{array}{ccc}
0, & 0, & 0 \\
0, & 0, & 0 \\
\frac{13}{24 h}, & \frac{1}{8}, & \frac{1}{24}
\end{array}\right),\left(\begin{array}{ccc}
0, & 0, & 0 \\
0, & 0, & 0 \\
\frac{13}{24 h}, & \frac{-1}{8}, & \frac{1}{24}
\end{array}\right),\left(\begin{array}{ccc}
1, & 0, & 0 \\
0, & 1, & 0 \\
\frac{-7}{3 h}, & 0, & 0
\end{array}\right)\right\} ;
$$

North boundary (neighbors $2,3,4,5$ ):

$$
\left\{\left(\begin{array}{ccc}
0, & 0, & 0 \\
0, & 0, & 0 \\
\frac{-13}{24 h}, & \frac{-1}{8}, & \frac{1}{24}
\end{array}\right),\left(\begin{array}{ccc}
0, & 0, & 0 \\
0, & 0, & 0 \\
\frac{-5}{4 h}, & 0, & \frac{-1}{3}
\end{array}\right),\left(\begin{array}{ccc}
0, & 0, & 0 \\
0, & 0, & 0 \\
\frac{-13}{24 h}, & \frac{1}{8}, & \frac{1}{24}
\end{array}\right),\left(\begin{array}{ccc}
1, & 0, & 0 \\
0, & 1, & 0 \\
\frac{7}{3 h}, & 0, & 0
\end{array}\right)\right\} ;
$$


East boundary (neighbors 1,2,3,5):

$$
\left\{\left(\begin{array}{ccc}
0, & 0, & 0 \\
\frac{-13}{24 h}, & \frac{1}{24}, & \frac{1}{8} \\
0, & 0, & 0
\end{array}\right),\left(\begin{array}{ccc}
0, & 0, & 0 \\
\frac{-5}{4 h}, & \frac{-1}{3}, & 0 \\
0, & 0, & 0
\end{array}\right),\left(\begin{array}{ccc}
0, & 0, & 0 \\
\frac{-13}{24 h}, & \frac{1}{24}, & \frac{-1}{8} \\
0, & 0, & 0
\end{array}\right),\left(\begin{array}{ccc}
1, & 0, & 0 \\
\frac{7}{3 h}, & 0, & 0 \\
0, & 0, & 1
\end{array}\right)\right\} ;
$$

West boundary (neighbors 1,3,4,5):

$$
\left\{\left(\begin{array}{ccc}
0, & 0, & 0 \\
\frac{13}{24 h}, & \frac{1}{24}, & \frac{-1}{8} \\
0, & 0, & 0
\end{array}\right),\left(\begin{array}{ccc}
0, & 0, & 0 \\
\frac{13}{24 h}, & \frac{1}{24}, & \frac{1}{8} \\
0, & 0, & 0
\end{array}\right),\left(\begin{array}{ccc}
0, & 0, & 0 \\
\frac{5}{4 h}, & \frac{-1}{3}, & 0 \\
0, & 0, & 0
\end{array}\right),\left(\begin{array}{ccc}
1, & 0, & 0 \\
\frac{-7}{3 h}, & 0, & 0 \\
0, & 0, & 1
\end{array}\right)\right\} .
$$

Corresponding errors in components of first-order derivatives are of order $\frac{h^{5}}{6 ! 3} U_{60}, \frac{h^{5}}{6 ! 3} U_{51}$.

Triangular case $k=2$ boundary nodes. Optimal weights (matrices $W$ ) are: South boundary (neighbors $1,2,4,5)$ :

$$
\begin{gathered}
W_{1}=\left(\begin{array}{llllll}
0, & 0, & 0, & 0, & 0, & 0 \\
0, & 0, & 0, & 0, & 0, & 0 \\
0, & 0, & \frac{1}{2}, & 0, & 0, & \frac{-h}{8} \\
0, & 0, & 0, & 0, & 0, & 0 \\
0, & 0, & 0, & 0, & \frac{1}{2}, & 0 \\
0, & 0, & 0, & 0, & 0, & 0
\end{array}\right), W_{2}=\left(\begin{array}{ccccccc}
0, & 0, & 0, & 0, & 0, & 0 \\
0, & 0, & 0, & 0, & 0, & 0 \\
0, & \frac{1}{16}, & \frac{1}{4}, & 0, & \frac{h}{16}, & 0 \\
0, & 0, & 0, & 0, & 0, & 0 \\
0, & 0, & 0, & \frac{-1}{8}, & \frac{1}{4}, & \frac{1}{8} \\
0, & 0, & 0, & 0, & 0, & 0
\end{array}\right), \\
W_{4}=\left(\begin{array}{cccccc}
0, & 0, & 0, & 0, & 0, & 0 \\
0, & 0, & 0, & 0, & 0, & 0 \\
0, & \frac{-1}{16}, & \frac{1}{4}, & 0, & \frac{-h}{16}, & 0 \\
0, & 0, & 0, & 0, & 0, & 0 \\
0, & 0, & 0, & \frac{1}{8}, & \frac{1}{4}, & \frac{-1}{8} \\
0, & 0, & 0, & 0, & 0, & 0
\end{array}\right), W_{5}=\left(\begin{array}{cccccc}
1, & 0, & 0, & 0, & 0, & 0 \\
0, & 1, & 0, & 0, & 0, & 0 \\
0, & 0, & 0, & \frac{h}{2}, & 0, & 0 \\
0, & 0, & 0, & 1, & 0, & 0 \\
0, & 0, & 0, & 0, & 0, & 0 \\
0, & 0, & 0, & 0, & 0, & 1
\end{array}\right) ;
\end{gathered}
$$

North boundary (neighbors 2,3,4,5):

$$
\begin{gathered}
W_{2}=\left(\begin{array}{cccccc}
0, & 0, & 0, & 0, & 0, & 0 \\
0, & 0, & 0, & 0, & 0, & 0 \\
0, & \frac{-1}{16}, & \frac{1}{4}, & 0, & \frac{h}{16}, & 0 \\
0, & 0, & 0, & 0, & 0, & 0 \\
0, & 0, & 0, & \frac{1}{8}, & \frac{1}{4}, & \frac{-1}{8} \\
0, & 0, & 0, & 0, & 0, & 0
\end{array}\right), W_{3}=\left(\begin{array}{ccccccc}
0, & 0, & 0, & 0, & 0, & 0 \\
0, & 0, & 0, & 0, & 0, & 0 \\
0, & 0, & \frac{1}{2}, & 0, & 0, & \frac{h}{8} \\
0, & 0, & 0, & 0, & 0, & 0 \\
0, & 0, & 0, & 0, & \frac{1}{2}, & 0 \\
0, & 0, & 0, & 0, & 0, & 0
\end{array}\right), \\
W_{4}=\left(\begin{array}{cccccc}
0, & 0, & 0, & 0, & 0, & 0 \\
0, & 0, & 0, & 0, & 0, & 0 \\
0, & \frac{1}{16}, & \frac{1}{4}, & 0, & \frac{-h}{16}, & 0 \\
0, & 0, & 0, & 0, & 0, & 0 \\
0, & 0, & 0, & \frac{-1}{8}, & \frac{1}{4}, & \frac{1}{8} \\
0, & 0, & 0, & 0, & 0, & 0
\end{array}\right), W_{5}=\left(\begin{array}{cccccc}
1, & 0, & 0, & 0, & 0, & 0 \\
0, & 1, & 0, & 0, & 0, & 0 \\
0, & 0, & 0, & \frac{-h}{2}, & 0, & 0 \\
0, & 0, & 0, & 1, & 0, & 0 \\
0, & 0, & 0, & 0, & 0, & 0 \\
0, & 0, & 0, & 0, & 0, & 1
\end{array}\right) ;
\end{gathered}
$$

East boundary (neighbors 1,2,3,5):

$$
W_{1}=\left(\begin{array}{cccccc}
0, & 0, & 0, & 0, & 0, & 0 \\
0, & \frac{1}{4}, & \frac{1}{16}, & 0, & \frac{-h}{16}, & 0 \\
0, & 0, & 0, & 0, & 0, & 0 \\
0, & 0, & 0, & \frac{1}{8}, & \frac{1}{4}, & \frac{-1}{8} \\
0, & 0, & 0, & 0, & 0, & 0 \\
0, & 0, & 0, & 0, & 0, & 0
\end{array}\right), W_{2}=\left(\begin{array}{cccccc}
0, & 0, & 0, & 0, & 0, & 0 \\
0, & \frac{1}{2}, & 0, & \frac{h}{8}, & 0, & 0 \\
0, & 0, & 0, & 0, & 0, & 0 \\
0, & 0, & 0, & 0, & 0, & 0 \\
0, & 0, & 0, & 0, & \frac{1}{2}, & 0 \\
0, & 0, & 0, & 0, & 0, & 0
\end{array}\right)
$$




$$
W_{3}=\left(\begin{array}{cccccc}
0, & 0, & 0, & 0, & 0, & 0 \\
0, & \frac{1}{4}, & \frac{-1}{16}, & 0, & \frac{h}{16}, & 0 \\
0, & 0, & 0, & 0, & 0, & 0 \\
0, & 0, & 0, & 0, & 0, & 0 \\
0, & 0, & 0, & \frac{-1}{8}, & \frac{1}{4}, & \frac{1}{8} \\
0, & 0, & 0, & 0, & 0, & 0
\end{array}\right), \quad W_{5}=\left(\begin{array}{cccccc}
1, & 0, & 0, & 0, & 0, & 0 \\
0, & 0, & 0, & 0, & 0, & \frac{-h}{2} \\
0, & 0, & 1, & 0, & 0, & 0 \\
0, & 0, & 0, & 1, & 0, & 0 \\
0, & 0, & 0, & 0, & 0, & 0 \\
0, & 0, & 0, & 0, & 0, & 1
\end{array}\right) ;
$$

West boundary (neighbors 1,3,4,5):

$$
\begin{gathered}
W_{1}=\left(\begin{array}{cccccc}
0, & 0, & 0, & 0, & 0, & 0 \\
0, & \frac{1}{4}, & \frac{-1}{16}, & 0, & \frac{h}{16}, & 0 \\
0, & 0, & 0, & 0, & 0, & 0 \\
0, & 0, & 0, & 0, & 0, & 0 \\
0, & 0, & 0, & \frac{-1}{8}, & \frac{1}{4}, & \frac{1}{8} \\
0, & 0, & 0, & 0, & 0, & 0
\end{array}\right), W_{3}=\left(\begin{array}{cccccc}
0, & 0, & 0, & 0, & 0, & 0 \\
0, & \frac{1}{4}, & \frac{1}{16}, & 0, & \frac{-h}{16}, & 0 \\
0, & 0, & 0, & 0, & 0, & 0 \\
0, & 0, & 0, & 0, & 0, & 0 \\
0, & 0, & 0, & \frac{1}{8}, & \frac{1}{4}, & \frac{-1}{8} \\
0, & 0, & 0, & 0, & 0, & 0
\end{array}\right), \\
W_{4}=\left(\begin{array}{llllll}
0, & 0, & 0, & 0, & 0, & 0 \\
0, & \frac{1}{2}, & 0, & \frac{-h}{8}, & 0, & 0 \\
0, & 0, & 0, & 0, & 0, & 0 \\
0, & 0, & 0, & 0, & \frac{1}{2}, & 0 \\
0, & 0, & 0, & 0, & 0, & 0 \\
0, & 0, & 0, & 0, & 0, & 0
\end{array}\right), W_{5}=\left(\begin{array}{cccccc}
1, & 0, & 0, & 0, & 0, & 0 \\
0, & 0, & 0, & 0, & 0, & \frac{h}{2} \\
0, & 0, & 1, & 0, & 0, & 0 \\
0, & 0, & 0, & 1, & 0, & 0 \\
0, & 0, & 0, & 0, & 0, & 0 \\
0, & 0, & 0, & 0, & 0, & 1
\end{array}\right) .
\end{gathered}
$$

Corresponding errors are of order $\frac{h^{7}}{7 ! 2} U_{80}$ for first order derivatives and of order $\frac{h^{3}}{6} U_{41}$ for derivative $\partial_{x} \partial_{y}$.

Full case $k=2$ boundary nodes. Optimal weights (matrices $W$ ) are:

South boundary (neighbors $1,2,4,5$ ):

$$
\begin{aligned}
W_{1} & =\left(\begin{array}{cccccc}
0, & 0, & 0, & 0, & 0, & 0 \\
0, & 0, & 0, & 0, & 0, & 0 \\
\frac{45}{32 h}, & 0, & \frac{-113}{224}, & 0, & 0, & \frac{11 h}{224} \\
0, & 0, & 0, & 0, & 0, & 0 \\
0, & \frac{5}{8 h}, & 0, & 0, & \frac{-1}{8}, & 0 \\
0, & 0, & 0, & 0, & 0, & 0
\end{array}\right), \\
W_{2} & =\left(\begin{array}{cccccc}
0, & 0, & 0, & 0, & 0, & 0 \\
0, & 0, & 0, & 0, & 0, & 0 \\
\frac{213}{448 h}, & \frac{137}{896}, & \frac{11}{224}, & \frac{11 h}{256 \cdot 7}, & \frac{h}{112}, & \frac{-11 h}{256 \cdot 7} \\
0, & 0, & 0, & 0, & 0, & 0 \\
\frac{-105}{32 h}, & \frac{-35}{32 h}, & \frac{-5}{16 h}, & \frac{-3}{64}, & \frac{-1}{16}, & \frac{3}{64} \\
0, & 0, & 0, & 0, & 0, & 0
\end{array}\right), \\
W_{4} & =\left(\begin{array}{cccccc}
0, & 0, & 0, & 0, & 0, & 0 \\
0, & 0, & 0, & 0, & 0, & 0 \\
\frac{213}{448 h}, & \frac{-137}{896}, & \frac{11}{224}, & \frac{11 h}{256 \cdot 7}, & \frac{-h}{12}, & \frac{-11 h}{256 \cdot 7} \\
0, & 0, & 0, & 0, & 0, & 0 \\
\frac{105}{32 h^{2}}, & \frac{-35}{32 h}, & \frac{5}{16 h}, & \frac{3}{64}, & \frac{-1}{16}, & \frac{-3}{64} \\
0, & 0, & 0, & 0, & 0, & 0
\end{array}\right), \\
W_{5} & =\left(\begin{array}{cccccc}
1, & 0, & 0, & 0, & 0, & 0 \\
0, & 1, & 0, & 0, & 0, & 0 \\
\frac{-33}{14 h}, & 0, & 0, & \frac{3 h}{56}, & 0, & 0 \\
0, & 0, & 0, & 1, & 0, & 0 \\
0, & \frac{-5}{h}, & 0, & 0, & 0, & 0 \\
0, & 0, & 0, & 0, & 0, & 1
\end{array}\right) ;
\end{aligned}
$$


North boundary (neighbors 2,3,4,5):

$$
\begin{aligned}
W_{2} & =\left(\begin{array}{cccccc}
0, & 0, & 0, & 0, & 0, & 0 \\
0, & 0, & 0, & 0, & 0, & 0 \\
\frac{-213}{448 h}, & \frac{-137}{896}, & \frac{11}{224}, & \frac{-11 h}{256 \cdot 7}, & \frac{h}{112}, & \frac{11 h}{256 \cdot 7} \\
0, & 0, & 0, & 0, & 0, & 0 \\
\frac{105}{32 h^{2}}, & \frac{35}{32 h}, & \frac{-5}{16 h}, & \frac{3}{64}, & \frac{-1}{16}, & \frac{-3}{64} \\
0, & 0, & 0, & 0, & 0, & 0
\end{array}\right), \\
W_{3} & =\left(\begin{array}{cccccc}
0, & 0, & 0, & 0, & 0, & 0 \\
0, & 0, & 0, & 0, & 0, & 0 \\
\frac{-45}{32 h}, & 0, & \frac{-113}{224}, & 0, & 0, & \frac{-11 h}{224} \\
0, & 0, & 0, & 0, & 0, & 0 \\
0, & \frac{-5}{8 h}, & 0, & 0, & \frac{-1}{8}, & 0 \\
0, & 0, & 0, & 0, & 0, & 0
\end{array}\right), \\
W_{4} & =\left(\begin{array}{ccccccc}
0, & 0, & 0, & 0, & 0, & 0 \\
0, & 0, & 0, & 0, & 0, & 0 \\
\frac{213}{448 h}, & \frac{137}{896}, & \frac{11}{224}, & \frac{-11 h}{256.7}, & \frac{-h}{112}, & \frac{11 h}{256 \cdot 7} \\
0, & 0, & 0, & 0, & 0, & 0 \\
\frac{-105}{32 h^{2}}, & \frac{35}{32 h}, & \frac{5}{16 h}, & \frac{-3}{64}, & \frac{-1}{16}, & \frac{3}{64} \\
0, & 0, & 0, & 0, & 0, & 0
\end{array}\right), \\
W_{5} & =\left(\begin{array}{cccccc}
1, & 0, & 0, & 0, & 0, & 0 \\
0, & 1, & 0, & 0, & 0, & 0 \\
\frac{33}{14 h}, & 0, & 0, & \frac{-3 h}{56}, & 0, & 0 \\
0, & 0, & 0, & 1, & 0, & 0 \\
0, & \frac{5}{h}, & 0, & 0, & 0, & 0 \\
0, & 0, & 0, & 0, & 0, & 1
\end{array}\right) ;
\end{aligned}
$$

East boundary (neighbors $1,2,3,5$ ):

$$
\begin{aligned}
& W_{1}=\left(\begin{array}{cccccc}
0, & 0, & 0, & 0, & 0, & 0 \\
\frac{-213}{448 h}, & \frac{11}{224}, & \frac{137}{896}, & \frac{11 h}{256 \cdot 7}, & \frac{-h}{112}, & \frac{-11 h}{256 \cdot 7} \\
0, & 0, & 0, & 0, & 0, & 0 \\
0, & 0, & 0, & 0, & 0, & 0 \\
\frac{-105}{32 h^{2}}, & \frac{5}{16 h}, & \frac{35}{32 h}, & \frac{3}{64}, & \frac{-1}{16}, & \frac{-3}{64} \\
0, & 0, & 0, & 0, & 0, & 0
\end{array}\right), \\
& W_{2}=\left(\begin{array}{cccccc}
0, & 0, & 0, & 0, & 0, & 0 \\
\frac{-45}{32 h}, & \frac{-113}{224}, & 0, & \frac{-11 h}{224}, & 0, & 0 \\
0, & 0, & 0, & 0, & 0, & 0 \\
0, & 0, & 0, & 0, & 0, & 0 \\
0, & 0, & \frac{-5}{8 h}, & 0, & \frac{-1}{8}, & 0 \\
0, & 0, & 0, & 0, & 0, & 0
\end{array}\right) \text {, } \\
& W_{3}=\left(\begin{array}{cccccc}
0, & 0, & 0, & 0, & 0, & 0 \\
\frac{-213}{48 h h}, & \frac{11}{224}, & \frac{-137}{896}, & \frac{11 h}{256 \cdot 7}, & \frac{h}{112}, & \frac{-11 h}{256 \cdot 7} \\
0, & 0, & 0, & 0, & 0, & 0 \\
0, & 0, & 0, & 0, & 0, & 0 \\
\frac{105}{305}, & \frac{-5}{16 h}, & \frac{35}{32 h}, & \frac{-3}{64}, & \frac{-1}{16}, & \frac{3}{64} \\
0, & 0, & 0, & 0, & 0, & 0
\end{array}\right), \\
& W_{5}=\left(\begin{array}{cccccc}
1, & 0, & 0, & 0, & 0, & 0 \\
0, & 1, & 0, & 0, & 0, & 0 \\
\frac{33}{14 h}, & 0, & 0, & 0, & 0, & \frac{-3 h}{56} \\
0, & 0, & 0, & 1, & 0, & 0 \\
0, & 0, & \frac{5}{h}, & 0, & 0, & 0 \\
0, & 0, & 0, & 0, & 0, & 1
\end{array}\right) ;
\end{aligned}
$$



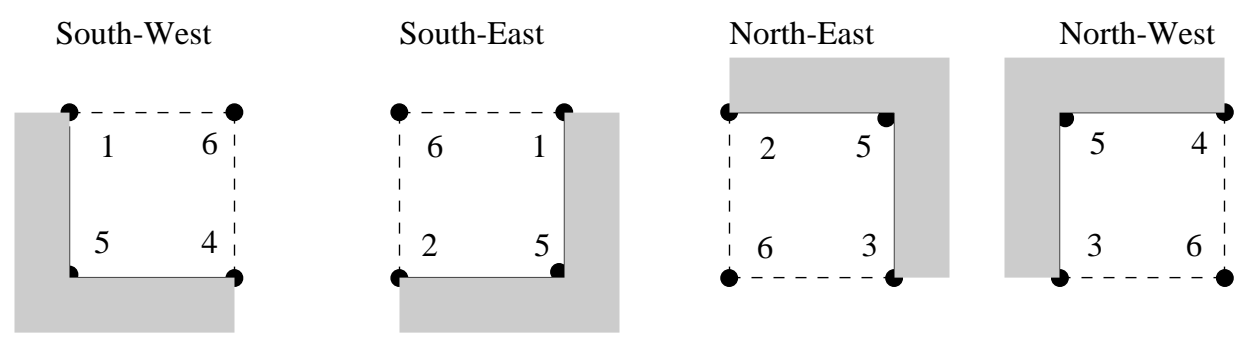

FiguRe 6.3.1

West boundary (neighbors 1,3,4,5):

$$
\begin{aligned}
W_{1} & =\left(\begin{array}{cccccc}
0, & 0, & 0, & 0, & 0, & 0 \\
\frac{213}{448 h}, & \frac{11}{224}, & \frac{-137}{896}, & \frac{-11 h}{256 \cdot 7}, & \frac{-h}{12}, & \frac{11 h}{256 \cdot 7} \\
0, & 0, & 0, & 0, & 0, & 0 \\
0, & 0, & 0, & 0, & 0, & 0 \\
\frac{105}{32 h^{2}}, & \frac{5}{16 h}, & \frac{-35}{32 h}, & \frac{-3}{64}, & \frac{-1}{16}, & \frac{3}{64} \\
0, & 0, & 0, & 0, & 0, & 0
\end{array}\right), \\
W_{3} & =\left(\begin{array}{cccccc}
0, & 0, & 0, & 0, & 0, & 0 \\
\frac{213}{448 h}, & \frac{11}{224}, & \frac{137}{896}, & \frac{-11 h}{256 \cdot 7}, & \frac{h}{112}, & \frac{11 h}{256 \cdot 7} \\
0, & 0, & 0, & 0, & 0, & 0 \\
0, & 0, & 0, & 0, & 0, & 0 \\
\frac{-105}{32 h}, & \frac{-5}{16 h}, & \frac{-35}{32 h}, & \frac{3}{64}, & \frac{-1}{16}, & \frac{-3}{64} \\
0, & 0, & 0, & 0, & 0, & 0
\end{array}\right), \\
W_{4} & =\left(\begin{array}{cccccc}
0, & 0, & 0, & 0, & 0, & 0 \\
\frac{45}{32 h}, & \frac{-113}{224}, & 0, & \frac{11 h}{224}, & 0, & 0 \\
0, & 0, & 0, & 0, & 0, & 0 \\
0, & 0, & 0, & 0, & 0, & 0 \\
0, & 0, & \frac{5}{8 h}, & 0, & \frac{-1}{8}, & 0 \\
0, & 0, & 0, & 0, & 0, & 0
\end{array}\right), \\
W_{5} & =\left(\begin{array}{cccccc}
1, & 0, & 0, & 0, & 0, & 0 \\
\frac{-33}{14 h}, & 0, & 0, & 0, & 0, & \frac{3 h}{56} \\
0, & 1, & 0, & 0, & 0, & 0 \\
0, & 0, & 0, & 1, & 0, & 0 \\
0, & 0, & \frac{-5}{h}, & 0, & 0, & 0 \\
0, & 0, & 0, & 0, & 0, & 1
\end{array}\right) .
\end{aligned}
$$

Corresponding errors are of order $\frac{38 h^{9}}{7 ! 8 !} U_{82}$ for first order derivatives and of order $\frac{h^{7}}{8 ! 3} U_{81}$ for derivative $\partial_{x} \partial_{y}$.

6.3. Optimal weights at corners. At each corner node we define the following nodes as neighbors: ennumeration of neighbors is shown in Figure 6.3.1. Each corner has 3 neighbors, since otherwise in the triangular case there is linear dependence between rows of the linear operator when $k=2$. For $k \leq 1$, these corner nodes do not play any role since all derivatives are known.

The value of $U_{10}$ is known because we can differentiate the given function $\varphi$ along $x$-direction and in the same way as the $U_{01}$ derivative. The situation changes for $k=2$ since the $U_{11}$ derivative cannot be found from the boundary data. Thus for $k=2$ the fifth lines of $W_{l}$ matrices (corresponding to the $\partial_{x} \partial_{y}$ derivative) are important. 
Triangular case $k=2$ boundary nodes. Optimal weights (matrices $W$ ) are: South-West corner (neighbors 1,4,5,6):

$$
\begin{aligned}
W_{1} & =\left(\begin{array}{llllll}
0, & 0, & 0, & 0, & 0, & 0 \\
0, & 0, & 0, & 0, & 0, & 0 \\
0, & 0, & 0, & 0, & 0, & 0 \\
0, & 0, & 0, & 0, & 0, & 0 \\
0, & 0, & 0, & -1, & -1, & 0 \\
0, & 0, & 0, & 0, & 0, & 0
\end{array}\right), W_{4}=\left(\begin{array}{ccccccc}
0, & 0, & 0, & 0, & 0, & 0 \\
0, & 0, & 0, & 0, & 0, & 0 \\
0, & 0, & 0, & 0, & 0, & 0 \\
0, & 0, & 0, & 0, & 0, & 0 \\
0, & 0, & 0, & 1, & 1, & 0 \\
0, & 0, & 0, & 0, & 0, & 0
\end{array}\right), \\
W_{5} & =\left(\begin{array}{llllll}
1, & 0, & 0, & 0, & 0, & 0 \\
0, & 1, & 0, & 0, & 0, & 0 \\
0, & 0, & 1, & 0, & 0, & 0 \\
0, & 0, & 0, & 1, & 0, & 0 \\
0, & 0, & 0, & 1, & 0, & 0 \\
0, & 0, & 0, & 0, & 0, & 1
\end{array}\right), W_{6}=\left(\begin{array}{cccccc}
0, & 0, & 0, & 0, & 0, & 0 \\
0, & 0, & 0, & 0, & 0, & 0 \\
0, & 0, & 0, & 0, & 0, & 0 \\
0, & 0, & 0, & 0, & 0, & 0 \\
0, & 0, & 0, & -1, & 1, & 0 \\
0, & 0, & 0, & 0, & 0, & 0
\end{array}\right) ;
\end{aligned}
$$

South-East corner (neighbors 1,2,5,6):

$$
\begin{aligned}
W_{1} & =\left(\begin{array}{llllll}
0, & 0, & 0, & 0, & 0, & 0 \\
0, & 0, & 0, & 0, & 0, & 0 \\
0, & 0, & 0, & 0, & 0, & 0 \\
0, & 0, & 0, & 0, & 0, & 0 \\
0, & 0, & 0, & 1, & -1, & 0 \\
0, & 0, & 0, & 0, & 0, & 0
\end{array}\right), W_{2}=\left(\begin{array}{cccccc}
0, & 0, & 0, & 0, & 0, & 0 \\
0, & 0, & 0, & 0, & 0, & 0 \\
0, & 0, & 0, & 0, & 0, & 0 \\
0, & 0, & 0, & 0, & 0, & 0 \\
0, & 0, & 0, & -1, & 1, & 0 \\
0, & 0, & 0, & 0, & 0, & 0
\end{array}\right), \\
W_{5} & =\left(\begin{array}{lllllll}
1, & 0, & 0, & 0, & 0, & 0 \\
0, & 1, & 0, & 0, & 0, & 0 \\
0, & 0, & 1, & 0, & 0, & 0 \\
0, & 0, & 0, & 1, & 0, & 0 \\
0, & 0, & 0, & -1, & 0, & 0 \\
0, & 0, & 0, & 0, & 0, & 1
\end{array}\right), W_{6}=\left(\begin{array}{cccccc}
0, & 0, & 0, & 0, & 0, & 0 \\
0, & 0, & 0, & 0, & 0, & 0 \\
0, & 0, & 0, & 0, & 0, & 0 \\
0, & 0, & 0, & 0, & 0, & 0 \\
0, & 0, & 0, & 1, & 1, & 0 \\
0, & 0, & 0, & 0, & 0, & 0
\end{array}\right) ;
\end{aligned}
$$

North-East corner (neighbors 2,3,5,6):

$$
\begin{aligned}
W_{2} & =\left(\begin{array}{llllll}
0, & 0, & 0, & 0, & 0, & 0 \\
0, & 0, & 0, & 0, & 0, & 0 \\
0, & 0, & 0, & 0, & 0, & 0 \\
0, & 0, & 0, & 0, & 0, & 0 \\
0, & 0, & 0, & 1, & 1, & 0 \\
0, & 0, & 0, & 0, & 0, & 0
\end{array}\right), W_{3}=\left(\begin{array}{ccccccc}
0, & 0, & 0, & 0, & 0, & 0 \\
0, & 0, & 0, & 0, & 0, & 0 \\
0, & 0, & 0, & 0, & 0, & 0 \\
0, & 0, & 0, & 0, & 0, & 0 \\
0, & 0, & 0, & -1, & -1, & 0 \\
0, & 0, & 0, & 0, & 0, & 0
\end{array}\right), \\
W_{5} & =\left(\begin{array}{llllll}
1, & 0, & 0, & 0, & 0, & 0 \\
0, & 1, & 0, & 0, & 0, & 0 \\
0, & 0, & 1, & 0, & 0, & 0 \\
0, & 0, & 0, & 1, & 0, & 0 \\
0, & 0, & 0, & 1, & 0, & 0 \\
0, & 0, & 0, & 0, & 0, & 1
\end{array}\right), W_{6}=\left(\begin{array}{cccccc}
0, & 0, & 0, & 0, & 0, & 0 \\
0, & 0, & 0, & 0, & 0, & 0 \\
0, & 0, & 0, & 0, & 0, & 0 \\
0, & 0, & 0, & 0, & 0, & 0 \\
0, & 0, & 0, & -1, & 1, & 0 \\
0, & 0, & 0, & 0, & 0, & 0
\end{array}\right) ;
\end{aligned}
$$

North-West corner (neighbors $3,4,5,6$ ):

$$
W_{3}=\left(\begin{array}{cccccc}
0, & 0, & 0, & 0, & 0, & 0 \\
0, & 0, & 0, & 0, & 0, & 0 \\
0, & 0, & 0, & 0, & 0, & 0 \\
0, & 0, & 0, & 0, & 0, & 0 \\
0, & 0, & 0, & 1, & -1, & 0 \\
0, & 0, & 0, & 0, & 0, & 0
\end{array}\right), W_{4}=\left(\begin{array}{cccccc}
0, & 0, & 0, & 0, & 0, & 0 \\
0, & 0, & 0, & 0, & 0, & 0 \\
0, & 0, & 0, & 0, & 0, & 0 \\
0, & 0, & 0, & 0, & 0, & 0 \\
0, & 0, & 0, & -1, & 1, & 0 \\
0, & 0, & 0, & 0, & 0, & 0
\end{array}\right),
$$




$$
W_{5}=\left(\begin{array}{cccccc}
1, & 0, & 0, & 0, & 0, & 0 \\
0, & 1, & 0, & 0, & 0, & 0 \\
0, & 0, & 1, & 0, & 0, & 0 \\
0, & 0, & 0, & 1, & 0, & 0 \\
0, & 0, & 0, & -1, & 0, & 0 \\
0, & 0, & 0, & 0, & 0, & 1
\end{array}\right), \quad W_{6}=\left(\begin{array}{cccccc}
0, & 0, & 0, & 0, & 0, & 0 \\
0, & 0, & 0, & 0, & 0, & 0 \\
0, & 0, & 0, & 0, & 0, & 0 \\
0, & 0, & 0, & 0, & 0, & 0 \\
0, & 0, & 0, & 1, & 1, & 0 \\
0, & 0, & 0, & 0, & 0, & 0
\end{array}\right)
$$

Corresponding errors are of order $\frac{h^{3}}{3} U_{41}$ for derivative $\partial_{x} \partial_{y}$.

Full case $k=2$ boundary nodes. Optimal weights (matrices $W$ ) are:

South-West corner (neighbors $1,4,5,6$ ):

$$
\begin{aligned}
W_{1} & =\left(\begin{array}{cccccc}
0, & 0, & 0, & 0, & 0, & 0 \\
0, & 0, & 0, & 0, & 0, & 0 \\
0, & 0, & 0, & 0, & 0, & 0 \\
0, & 0, & 0, & 0, & 0, & 0 \\
\frac{24}{h^{2}}, & \frac{7}{h}, & \frac{-7}{h}, & 0, & -1, & 0 \\
0, & 0, & 0, & 0, & 0, & 0
\end{array}\right), W_{4}=\left(\begin{array}{ccccccc}
0, & 0, & 0, & 0, & 0, & 0 \\
0, & 0, & 0, & 0, & 0, & 0 \\
0, & 0, & 0, & 0, & 0, & 0 \\
0, & 0, & 0, & 0, & 0, & 0 \\
\frac{24}{h^{2}}, & \frac{-7}{h}, & \frac{7}{h}, & 0, & -1, & 0 \\
0, & 0, & 0, & 0, & 0, & 0
\end{array}\right), \\
W_{5} & =\left(\begin{array}{cccccc}
1, & 0, & 0, & 0, & 0, & 0 \\
0, & 1, & 0, & 0, & 0, & 0 \\
0, & 0, & 1, & 0, & 0, & 0 \\
0, & 0, & 0, & 1, & 0, & 0 \\
\frac{-24}{h^{2}}, & \frac{-7}{h}, & \frac{-7}{h}, & 0, & 0, & 0 \\
0, & 0, & 0, & 0, & 0, & 1
\end{array}\right), W_{6}=\left(\begin{array}{cccccc}
0, & 0, & 0, & 0, & 0, & 0 \\
0, & 0, & 0, & 0, & 0, & 0 \\
0, & 0, & 0, & 0, & 0, & 0 \\
0, & 0, & 0, & 0, & 0, & 0 \\
\frac{-24}{h^{2}}, & \frac{7}{h}, & \frac{7}{h}, & 0, & -1, & 0 \\
0, & 0, & 0, & 0, & 0, & 0
\end{array}\right) ;
\end{aligned}
$$

South-East corner (neighbors 1,2,5,6):

$$
\begin{gathered}
W_{1}=\left(\begin{array}{cccccc}
0, & 0, & 0, & 0, & 0, & 0 \\
0, & 0, & 0, & 0, & 0, & 0 \\
0, & 0, & 0, & 0, & 0, & 0 \\
0, & 0, & 0, & 0, & 0, & 0 \\
\frac{-24}{h^{2}}, & \frac{7}{h}, & \frac{7}{h}, & 0, & -1, & 0 \\
0, & 0, & 0, & 0, & 0, & 0
\end{array}\right), W_{2}=\left(\begin{array}{ccccccc}
0, & 0, & 0, & 0, & 0, & 0 \\
0, & 0, & 0, & 0, & 0, & 0 \\
0, & 0, & 0, & 0, & 0, & 0 \\
0, & 0, & 0, & 0, & 0, & 0 \\
\frac{-24}{h^{2}}, & \frac{-7}{h}, & \frac{-7}{h}, & 0, & -1, & 0 \\
0, & 0, & 0, & 0, & 0, & 0
\end{array}\right), \\
W_{5}=\left(\begin{array}{cccccc}
1, & 0, & 0, & 0, & 0, & 0 \\
0, & 1, & 0, & 0, & 0, & 0 \\
0, & 0, & 1, & 0, & 0, & 0 \\
0, & 0, & 0, & 1, & 0, & 0 \\
\frac{24}{h^{2}} & \frac{-7}{h}, & \frac{7}{h}, & 0, & 0, & 0 \\
0, & 0, & 0, & 0, & 0, & 1
\end{array}\right), W_{6}=\left(\begin{array}{cccccc}
0, & 0, & 0, & 0, & 0, & 0 \\
0, & 0, & 0, & 0, & 0, & 0 \\
0, & 0, & 0, & 0, & 0, & 0 \\
0, & 0, & 0, & 0, & 0, & 0 \\
\frac{24}{h^{2}} & \frac{7}{h}, & \frac{-7}{h} & 0, & -1, & 0 \\
0, & 0, & 0, & 0, & 0, & 0
\end{array}\right) ;
\end{gathered}
$$

North-East corner (neighbors 2,3,5,6):

$$
\begin{aligned}
& W_{2}=\left(\begin{array}{cccccc}
0, & 0, & 0, & 0, & 0, & 0 \\
0, & 0, & 0, & 0, & 0, & 0 \\
0, & 0, & 0, & 0, & 0, & 0 \\
0, & 0, & 0, & 0, & 0, & 0 \\
\frac{24}{h^{2}} & \frac{7}{h}, & \frac{-7}{h}, & 0, & -1, & 0 \\
0, & 0, & 0, & 0, & 0, & 0
\end{array}\right), \quad W_{3}=\left(\begin{array}{cccccc}
0, & 0, & 0, & 0, & 0, & 0 \\
0, & 0, & 0, & 0, & 0, & 0 \\
0, & 0, & 0, & 0, & 0, & 0 \\
0, & 0, & 0, & 0, & 0, & 0 \\
\frac{24}{h^{2}}, & \frac{-7}{h}, & \frac{7}{h}, & 0, & -1, & 0 \\
0, & 0, & 0, & 0, & 0, & 0
\end{array}\right), \\
& W_{5}=\left(\begin{array}{cccccc}
1, & 0, & 0, & 0, & 0, & 0 \\
0, & 1, & 0, & 0, & 0, & 0 \\
0, & 0, & 1, & 0, & 0, & 0 \\
0, & 0, & 0, & 1, & 0, & 0 \\
\frac{-24}{h^{2}}, & \frac{7}{h}, & \frac{7}{h}, & 0, & 0, & 0 \\
0, & 0, & 0, & 0, & 0, & 1
\end{array}\right) \quad W_{6}=\left(\begin{array}{cccccc}
0, & 0, & 0, & 0, & 0, & 0 \\
0, & 0, & 0, & 0, & 0, & 0 \\
0, & 0, & 0, & 0, & 0, & 0 \\
0, & 0, & 0, & 0, & 0, & 0 \\
\frac{-24}{h^{2}}, & \frac{-7}{h}, & \frac{-7}{h}, & 0, & -1, & 0 \\
0, & 0, & 0, & 0, & 0, & 0
\end{array}\right) ;
\end{aligned}
$$


North-West corner (neighbors 3,4,5,6):

$$
\begin{gathered}
W_{3}=\left(\begin{array}{cccccc}
0, & 0, & 0, & 0, & 0, & 0 \\
0, & 0, & 0, & 0, & 0, & 0 \\
0, & 0, & 0, & 0, & 0, & 0 \\
0, & 0, & 0, & 0, & 0, & 0 \\
\frac{-24}{h^{2}}, & \frac{-7}{h}, & \frac{-7}{h}, & 0, & -1, & 0 \\
0, & 0, & 0, & 0, & 0, & 0
\end{array}\right), W_{4}=\left(\begin{array}{ccccccc}
0, & 0, & 0, & 0, & 0, & 0 \\
0, & 0, & 0, & 0, & 0, & 0 \\
0, & 0, & 0, & 0, & 0, & 0 \\
0, & 0, & 0, & 0, & 0, & 0 \\
\frac{-24}{h^{2}}, & \frac{7}{h}, & \frac{7}{h}, & 0, & -1, & 0 \\
0, & 0, & 0, & 0, & 0, & 0
\end{array}\right), \\
W_{5}=\left(\begin{array}{cccccccc}
1, & 0, & 0, & 0, & 0, & 0 \\
0, & 1, & 0, & 0, & 0, & 0 \\
0, & 0, & 1, & 0, & 0, & 0 \\
0, & 0, & 0, & 1, & 0, & 0 \\
\frac{24}{h^{2}}, & \frac{7}{h}, & \frac{-7}{h}, & 0, & 0, & 0 \\
0, & 0, & 0, & 0, & 0, & 1
\end{array}\right), W_{6}=\left(\begin{array}{cccccc}
0, & 0, & 0, & 0, & 0, & 0 \\
0, & 0, & 0, & 0, & 0, & 0 \\
0, & 0, & 0, & 0, & 0, & 0 \\
0, & 0, & 0, & 0, & 0, & 0 \\
\frac{24}{h^{2}}, & \frac{-7}{h}, & \frac{7}{h}, & 0, & -1, & 0 \\
0, & 0, & 0, & 0, & 0, & 0
\end{array}\right) .
\end{gathered}
$$

Corresponding errors are of order $\frac{h^{8}}{10 ! 8} U_{91}$ for derivative $\partial_{x} \partial_{y}$.

6.4. Completion operators. As was mentioned in subsection 4.2, the accuracy we obtain by recovering a 2-order jet by its neighbors (assuming the Whitney functional to be sufficiently small) is much higher than the accuracy that can be achieved by using only values. This means that by straightforward use of jets of order 2, we allow errors of order of $h^{3}$ and have no benefits from the high accuracy of coefficients of this jet. However, in this scheme we suggest that one can also recover the higher derivatives from neighboring jets of order 2. Their accuracy is balanced in terms of degrees of $h$.

Assume that jets of second order of a harmonic function are known at 5 points (forming our stencil). One can find matrices $Q_{l}, l=1,2,3,4,5$ of dimension $\operatorname{dim} \mathrm{Jet}_{11} \times \operatorname{dim} \mathrm{Jet}_{2}$, such that the linear combination $\sum_{1}^{5} Q_{l} U_{2}^{z_{l}}$ reconstructs jet of order 11 of the original function at the middle point. The following table shows unreducible errors in terms of orders $2-11$; here symbol $(2,1)$, for example, means an error in the term corresponding to $\partial_{x x} \partial_{y}$, etc.

$$
\begin{aligned}
(3,0) & \rightarrow \frac{h^{10}}{15 ! 2156} U_{13,0}, & (2,1) & \rightarrow \frac{7 h^{10}}{10 ! 1170} U_{12,1}, \\
(4,0) & \rightarrow o\left(h^{9}\right), & (3,1) & \rightarrow \frac{6 h^{8}}{11 !} U_{11,1}, \\
(5,0) & \rightarrow \frac{2 h^{8}}{11 !} U_{13,0}, & (4,1) & \rightarrow \frac{2 h^{8}}{11 !} U_{12,1}, \\
(6,0) & \rightarrow o\left(h^{8}\right), & (5,1) & \rightarrow \frac{120 h^{8}}{13 !} U_{13,1}, \\
(7,0) & \rightarrow \frac{h^{6}}{6 ! 1170} U_{13,0}, & (6,1) & \rightarrow \frac{h^{6}}{6 ! 1170} U_{12,1}, \\
(8,0) & \rightarrow o\left(h^{5}\right), & (7,1) & \rightarrow \frac{h^{4}}{5 ! 33} U_{11,1}, \\
(9,0) & \rightarrow \frac{4 h^{4}}{6 ! 33} U_{13,0}, & (8,1) & \rightarrow \frac{4 h^{4}}{6 ! 33} U_{12,1}, \\
(10,0) & \rightarrow o\left(h^{5}\right), & (9,1) & \rightarrow \frac{2 h^{4}}{5 ! 143} U_{13,1}, \\
(11,0) & \rightarrow \frac{7 h^{2}}{2340} U_{13,0}, & (10,1) & \rightarrow \frac{7 h^{2}}{2340} U_{12,1} .
\end{aligned}
$$


As an example of the completion matrix $Q_{1}$ for the full scheme $k=2$, lines from $(3,0)$ to $(10,1)$ are listed below (the previous lines from $(0,0)$ to $(0,2)$ are given by the corresponding $\left.W_{1}\right)$. The matrices have only 5 columns since by the equation $(2,0)$ components of formal solution are always equal to $(0,2)$ components, which means that only 5 coefficients are independent (that is $\operatorname{dim} \operatorname{Sol}_{2}^{z}=5$ at any internal node $z)$.

$$
Q_{1}=\left(\begin{array}{ccccc}
0, & -59 / 240 h^{2}, & 0, & 0, & 1 / 30 h \\
-231 / 32 h^{3}, & 0, & 883 / 480 h^{2}, & 1 / 480 h, & 0 \\
18 / h^{4}, & 0, & -57 / 16 h^{3}, & -3 / 16 h^{2}, & 0 \\
0, & -21 / 8 h^{3}, & 0, & 0, & 3 / 8 h^{2} \\
0, & 12 / h^{4}, & 0, & 0, & -3 / 2 h^{3} \\
0, & 0, & 12 / h^{4}, & 3 / 2 h^{3}, & 0 \\
-1575 / 2 h^{6}, & 0, & 1035 / 8 h^{5}, & 45 / 8 h^{4}, & 0 \\
0, & 135 / 2 h^{5}, & 0, & 0, & -15 / 2 h^{4} \\
-5775 / h^{7}, & -273 / h^{6}, & -5775 / 4 h^{6}, & 385 / 4 h^{5}, & 21 / h^{5} \\
10395 / 2 h^{7}, & 0, & -35492 / h^{6}, & -273 / 2 h^{5}, & 0 \\
-30240 / h^{8}, & 0, & 9450 / h^{7}, & 630 / h^{6}, & 0 \\
0, & 945 / h^{7}, & 0, & 0, & -315 / h^{6} \\
0, & -10080 / h^{8}, & 0, & 0, & 2520 / h^{7} \\
0, & 0, & -10080 / h^{8}, & -2520 / h^{7}, & 0 \\
4762800 / h^{10}, & 0, & -1077300 / h^{9}, & -56700 / h^{8}, & 0 \\
0, & -113400 / h^{9}, & 0, & 0, & 22680 / h^{8} \\
26195400 / h^{11}, & 914760 / h^{10}, & -6548850 / h^{10}, & -436590 / h^{9}, & -166320 / h^{9} \\
-13097700 / h^{11}, & 0, & 4698540 / h^{10}, & 457380 / h^{9}, & 0
\end{array}\right)
$$

6.5. Explanation of the tables. All calculations were performed with the Mathematica 2.2 program on an IBM RS6000 computer. For some values of parameters the resulting linear system was too large (more than $1000 \times 1000)$ and the program did not succeed in inverting such a matrix. This is marked by a $\star$ in the corresponding position of each table.

Dimensions of subspaces. The grid $G$ consists of $N^{2}$ nodes among which there are $4(N-1)$ boundaries and $(N-1)^{2}$ internal nodes. Dimension of the JEF space is $\# G \cdot \operatorname{dim}$ Jet. After imposing all restrictions from the differential equation, its corollaries and boundary conditions, we get the dimension of the subspace of global formal solutions. The results are represented in the following table, where $\operatorname{dim}_{1}$ is the dimension of $\mathrm{JEF}_{k}^{G}$, $\operatorname{dim}_{2}$ is the dimension of $\mathrm{SOL}_{k}^{G}$ when we consider true

TABLE 6.1

\begin{tabular}{|c|c|c|c|c|c|c|c|c|c|c|c|}
\multicolumn{4}{c}{$\operatorname{dim}_{1}$} & \multicolumn{4}{c}{$\operatorname{dim}_{2}$} & \multicolumn{5}{c|}{$\operatorname{dim}_{3}$} \\
\hline $\mathrm{N} \backslash \mathrm{k}$ & 0 & 1 & 2 & & 0 & 1 & 2 & & 0 & 1 & 2 \\
\hline 3 & 9 & 27 & 54 & & 1 & 7 & 17 & & 1 & 3 & 5 \\
\hline 5 & 25 & 75 & 150 & & 9 & 39 & 73 & & 9 & 27 & 45 \\
\hline 9 & 81 & 243 & 486 & & 49 & 175 & 305 & & 49 & 147 & 245 \\
\hline 17 & 289 & 867 & 1734 & & 225 & 735 & 1249 & & 225 & 675 & 1125 \\
\hline
\end{tabular}


boundary restrictions see (see subsection 4.3), and $\operatorname{dim}_{3}$ is the dimension of $\mathrm{SOL}_{k}^{G}$ when we consider all boundary jets fixed.

Discretization errors, Dirichlet problem. In Tables 6.2-6.21 we give results of the comparison of the solution of the discretized problem and the analytic one. Two types of solutions were used: $u(x, y)=x^{7}-21 x^{5} y^{2}+35 x^{3} y^{4}-7 x y^{6}$ and $u(x, y)=$ $e^{3 x} \sin 3 y$. The domain $\Omega=[0,1] \times[0,1]$. The first function takes values from $[-3,8]$ on $\Omega$ (approximately), and its first derivatives vary in intervals $[-20,20]$ and second in $[-170,170]$. The second function and its first and second derivatives have the range $[0,20],[-60,60],[-180,180]$. See pictures at the end.

Now we study only properties of the discretization scheme and not the boundary and corners effects. So as the boundary data we have taken the whole jets of true solution both on the boundary and at corners.

The precise results of our computations for full method with $k=2$ when the true solution has a form of a polynomial of degree 7 (see Tables 6.4 and 6.20) are explained by the fact that the scheme we use has a discretization error of order of tenth derivatives (see 5.1, 5.2, 5.3 full case, $k=2$ ). Hence it is precise for all harmonic polynomials of lower degree.

Maximal values in the right side, Dirichlet problem. In Tables 6.6-6.9 maximal absolute values of the right-hand side of the corresponding linear system of equations are given, as explained in subsection 6.1.

Discretization errors, Poisson problem. Discretization errors for a Poisson problem with $f=-16\left(x^{2}+y^{2}\right) \sin 4 x y$ are given in Tables 6.10-6.13. The same domain and grids are considered as in the Dirichlet case.

Maximal values in the right side, Poisson problem. In Tables 6.14-6.17 maximal absolute values of the right-hand side of the corresponding linear system of equations are given, as explained in subsection 6.2.

Multiscaling. Tables 6.18 and 6.19 contain information about condition numbers with and without multiscaling. The multiscaling effect on the right-hand sides is reflected in Tables 6.6-6.9 and 6.14-6.17 as mentioned above.

Dirichlet boundary data. Discretization errors and condition numbers for the scheme implemented with true boundary data (see subsection 4.3) are given in Tables 6.20-6.22. Note that in this case the dimensions of the corresponding objects are bigger; see column $2\left(\operatorname{dim}_{2}\right)$ in Table 6.1.

TABle 6.2. Dirichlet problem. Discretization errors in jet-form. Analytic solution: $x^{7}-21 x^{5} y^{2}+35 x^{3} y^{4}-7 x y^{6}$.

\begin{tabular}{|c|c|c|c|}
\hline$N \backslash \mathrm{k}$ & 0 & 1 & 2 \\
\hline 3 & $\{0.547\}$ & $\{0,0,3.3\}$ & $\{0,0,0,6.6,6.6\}$ \\
\hline 5 & $\{0.220\}$ & $\{0.027,0.29,0.92\}$ & $\{0.002,0.07,0.08,1.8,1.8\}$ \\
\hline 9 & $\{0.059\}$ & $\{0.007,0.08,0.27\}$ & $\left\{8.10^{-4}, 0.02,0.02,0.5,0.5\right\}$ \\
\hline 17 & $\{0.015\}$ & $\{0.002,0.02,0.07\}$ & $\star$ \\
\hline
\end{tabular}


TABle 6.3. Dirichlet problem. Discretization errors in jet-form. Analytic solution: $e^{3 x} \sin 3 y$. Triangular method, boundary jets fixed.

\begin{tabular}{|c|c|c|c|}
\hline $\mathrm{N} \backslash \mathrm{k}$ & 0 & 1 & 2 \\
\hline 3 & $\{0.95\}$ & $\left\{2.810^{-3}, 2.8,0.2\right\}$ & $\left\{1.10^{-6}, 8.510^{-3}, 6.10^{-4}, 8.5,0.6\right\}$ \\
\hline 5 & $\{0.3\}$ & $\{0.03,0.89,0.36\}$ & $\left\{2.10^{-3}, 0.09,0.07,2.67,1.\right\}$ \\
\hline 9 & $\{0.08\}$ & $\{0.0079,0.23,0.096\}$ & $\left\{7.710^{-4}, 0.02,0.02,0.7,0.3\right\}$ \\
\hline 17 & $\{0.02\}$ & $\{0.002,0.06,0.02\}$ & $\star$ \\
\hline
\end{tabular}

TABle 6.4. Dirichlet problem. Discretization errors in jet-form. Analytic solution: $x^{7}-21 x^{5} y^{2}+35 x^{3} y^{4}-7 x y^{6}$. Full method, boundary jets fixed.

\begin{tabular}{|c|c|c|c|}
\hline $\mathrm{N} \backslash \mathrm{k}$ & 0 & 1 & 2 \\
\hline 3 & $\{0.547\}$ & $\{0,0.026,0\}$ & $\{0,0,0,0,0\}$ \\
\hline 5 & $\{0.220\}$ & $\left\{1.10^{-5}, 4.10^{-4}, 3.10^{-5}\right\}$ & $\{0,0,0,0,0\}$ \\
\hline 9 & $\{0.059\}$ & $\left\{1.210^{-7}, 5.610^{-6}, 5.310^{-7}\right\}$ & $\{0,0,0,0,0\}$ \\
\hline 17 & $\{0.015\}$ & $\left\{1.110^{-9}, 8.710^{-8}, 8.710^{-9}\right\}$ & $\star$ \\
\hline
\end{tabular}

TABle 6.5. Dirichlet problem. Discretization errors in jet-form. Analytic solution: $e^{3 x} \sin 3 y$. Full method, boundary jets fixed.

\begin{tabular}{|c|c|c|c|}
\hline N $\backslash k$ & 0 & 1 & 2 \\
\hline 3 & $\{0.95\}$ & $\left\{2.810^{-3}, 5.110^{-2}, 3.510^{-3}\right\}$ & $\left\{1.210^{-6}, 4.210^{-5}, 2.910^{-5}, 0.026,2.10^{-4}\right\}$ \\
\hline 5 & $\{0.3\}$ & $\left\{7.10^{-5}, 1.210^{-3}, 1.310^{-3}\right\}$ & $\left\{1.210^{-6}, 3.310^{-4}, 7.710^{-7}, 9.10^{-4}, 2.210^{-5}\right\}$ \\
\hline 9 & $\{0.08\}$ & $\left\{2.110^{-6}, 4.610^{-5}, 3.410^{-5}\right\}$ & $\left\{6.10^{-19}, 2.310^{-9}, 4.910^{-9}, 1.610^{-4}, 1.10^{-7}\right\}$ \\
\hline 17 & $\{0.02\}$ & $\left\{4.010^{-5}, 9.010^{-7}, 6.410^{-7}\right\}$ & + \\
\hline
\end{tabular}

TABLE 6.6. Dirichlet problem. Maximal absolute values in right hand side, triangular method, the whole boundary jets are fixed.

True solution: $x^{7}-21 x^{5} y^{2}+35 x^{3} y^{4}-7 x y^{6}$.

\begin{tabular}{|c|c|c|c|c|c|c|c|}
\multicolumn{4}{c}{ without multiscaling } & \multicolumn{3}{c|}{ with multiscaling } \\
\hline $\mathrm{N} \backslash \mathrm{k}$ & 0 & 1 & 2 & & 0 & 1 & 2 \\
\hline 3 & 0.484 & 2.406 & 1.312 & & 0.484 & 0.547 & 7. \\
\hline 5 & 0.92 & 8.8 & 34.4 & & 0.92 & 3.2 & 1.177 \\
\hline 9 & 2.31 & 17.75 & 58.1 & & 2.31 & 5.03 & 0.242 \\
\hline 17 & 3.133 & 22.78 & 70.92 & & 3.133 & 5.02 & $\star$ \\
\hline
\end{tabular}


TABle 6.7. Dirichlet problem. Maximal absolute values in right hand side, triangular method, the whole boundary jets are fixed. True solution: $e^{3 x} \sin 3 y$.

\begin{tabular}{|c|c|c|c|c|c|c|c|}
\hline \multicolumn{3}{c}{ without multiscaling } & \multicolumn{4}{c|}{ with multiscaling } \\
\hline $\mathrm{N} \backslash \mathrm{k}$ & 0 & 1 & 2 & & 0 & 1 & 2 \\
\hline 3 & 5.42 & 16.25 & 48.7 & & 5.42 & 18.52 & 9.08 \\
\hline 5 & 5.01 & 18.14 & 54.4 & & 5.01 & 15.55 & 1.332 \\
\hline 9 & 5.01 & 24.37 & 73.1 & & 5.01 & 15.18 & 0.148 \\
\hline 17 & 5.01 & 27.29 & 81.87 & & 5.01 & 15.06 & $\star$ \\
\hline
\end{tabular}

TABLE 6.8. Dirichlet problem. Maximal absolute values in right hand side, full method, the whole boundary jets are fixed. True solution: $x^{7}-21 x^{5} y^{2}+35 x^{3} y^{4}-7 x y^{6}$.

\begin{tabular}{|c|c|c|c|c|c|c|c|}
\hline \multicolumn{3}{c}{ without multiscaling } & \multicolumn{4}{c|}{ with multiscaling } \\
\hline $\mathrm{N} \backslash \mathrm{k}$ & 0 & 1 & 2 & & 0 & 1 & 2 \\
\hline 3 & 0.484 & 0.875 & 5.25 & & 0.484 & 2.828 & 1.312 \\
\hline 5 & 0.92 & 9.46 & 169.1 & & 0.92 & 3.43 & 0.144 \\
\hline 9 & 2.31 & 30.28 & 2081 & & 2.31 & 11.36 & 0.0106 \\
\hline 17 & 3.133 & 71.4 & 13791 & & 3.133 & 51. & $\star$ \\
\hline
\end{tabular}

TABLE 6.9. Dirichlet problem. Maximal absolute values in right hand side, full method, the whole boundary jets are fixed. True solution: $e^{3 x} \sin 3 y$.

\begin{tabular}{|c|c|c|c|c|c|c|c|}
\hline \multicolumn{4}{c}{ without multiscaling } & \multicolumn{4}{c|}{ with multiscaling } \\
\hline $\mathrm{N} \backslash \mathrm{k}$ & 0 & 1 & 2 & & 0 & 1 & 2 \\
\hline 3 & 5.42 & 13.36 & 40.3 & & 5.42 & 18.72 & 0.593 \\
\hline 5 & 5.01 & 40.1 & 1895 & & 5.01 & 37.2 & 0.171 \\
\hline 9 & 5.01 & 90.2 & 9670 & & 5.01 & 53.7 & 0.021 \\
\hline 17 & 5.01 & 27.29 & 43300 & & 5.01 & 15.1 & $\star$ \\
\hline
\end{tabular}

TABle 6.10. Poisson equation. Discretization errors in jet-form. Analytic solution: $x^{7}-21 x^{5} y^{2}+35 x^{3} y^{4}-7 x y^{6}+\sin 4 x y$. Triangular method, boundary jets fixed.

\begin{tabular}{|c|c|c|c|}
\hline $\mathrm{N} \backslash \mathrm{k}$ & 0 & 1 & 2 \\
\hline 3 & $\{0.513\}$ & $\left\{2.10^{-5}, 0.18,3.1\right\}$ & $\left\{3.610^{-7}, 2.410^{-4}, 7.10^{-4}, 7.5,6.3\right\}$ \\
\hline 5 & $\{0.2\}$ & $\{0.027,0.35,0.88\}$ & $\{0.003,0.074,0.084,2.0,1.9\}$ \\
\hline 9 & $\{0.05\}$ & $\{0.007,0.09,0.26\}$ & $\left\{8.10^{-4}, 0.02,0.02,0.6,0.5\right\}$ \\
\hline 17 & $\{0.01\}$ & $\{0.002,0.02,0.07\}$ & $\star$ \\
\hline
\end{tabular}


Table 6.11. Poisson equation. Discretization errors in jet-form. Analytic solution: $e^{3 x} \sin 3 y+\sin 4 x y$. Triangular method, boundary jets fixed.

\begin{tabular}{|c|c|c|c|}
\hline $\mathrm{N} \backslash \mathrm{k}$ & 0 & 1 & 2 \\
\hline 3 & $\{0.98\}$ & $\left\{3.10^{-3}, 3.0,0.4\right\}$ & $\left\{1.610^{-6}, 8.710^{-3}, 0.001,9.5,0.9\right\}$ \\
\hline 5 & $\{0.3\}$ & $\{0.03,0.96,0.39\}$ & $\left\{2.10^{-3}, 0.1,0.07,2.87,1.1\right\}$ \\
\hline 9 & $\{0.08\}$ & $\{0.008,0.25,0.1\}$ & $\left\{8.710^{-4}, 0.027,0.018,0.75,0.3\right\}$ \\
\hline 17 & $\{0.02\}$ & $\{0.002,0.06,0.03\}$ & $\star$ \\
\hline
\end{tabular}

TABle 6.12. Poisson equation. Discretization errors in jet-form. Analytic solution: $x^{7}-21 x^{5} y^{2}+35 x^{3} y^{4}-7 x y^{6}+\sin 4 x y$. Full method, boundary jets fixed.

\begin{tabular}{|c|c|c|c|}
\hline $\mathrm{N} \backslash \mathrm{k}$ & 0 & 1 & 2 \\
\hline 3 & $\{0.513\}$ & $\left\{2.10^{-5}, 0.027,0.001\right\}$ & $\left\{3.610^{-7}, 3.610^{-7}, 5.710^{-6}, 7.610^{-3}, 4.10^{-5}\right\}$ \\
\hline 5 & $\{0.2\}$ & $\left\{2.10^{-5}, 6.10^{-4}, 2.10^{-4}\right\}$ & $\left\{2.410^{-6}, 6.10^{-6}, 1.510^{-5}, 1.510^{-5}, 2.10^{-5}\right\}$ \\
\hline 9 & $\{0.05\}$ & $\left\{4.810^{-7}, 1.10^{-5}, 7.910^{-6}\right\}$ & $\left\{7.410^{-9}, 4.810^{-8}, 9.210^{-8}, 2.10^{-5}, 2.10^{-7}\right\}$ \\
\hline 17 & $\{0.01\}$ & $\left\{9.10^{-9}, 2.610^{-7}, 1.810^{-7}\right\}$ & $\star$ \\
\hline
\end{tabular}

Table 6.13. Poisson equation. Discretization errors in jet-form. Analytic solution: $e^{3 x} \sin 3 y+\sin 4 x y$. Full method, boundary jets fixed.

\begin{tabular}{|c|c|c|c|}
\hline$N \backslash k$ & 0 & 1 & 2 \\
\hline 3 & $\{0.98\}$ & $\left\{3.10^{-3}, 0.05,2.510^{-3}\right\}$ & $\left\{1.610^{-6}, 4.10^{-5}, 9.10^{-6}, 0.03,2.10^{-4}\right\}$ \\
\hline 5 & $\{0.3\}$ & $\left\{9.10^{-5}, 2.10^{-3}, 1.10^{-3}\right\}$ & $\left\{3.410^{-6}, 7.410^{-6}, 2.10^{-5}, 2.10^{-3}, 4.310^{-5}\right\}$ \\
\hline 9 & $\{0.08\}$ & $\left\{2.610^{-6}, 5.10^{-5}, 4.10^{-5}\right\}$ & $\left\{7.610^{-9}, 4.710^{-8}, 9.10^{-8}, 2.10^{-5}, 3.210^{-7}\right\}$ \\
\hline 17 & $\{0.02\}$ & $\left\{4.910^{-8}, 1.10^{-6}, 7.710^{-7}\right\}$ & $\star$ \\
\hline
\end{tabular}

TABle 6.14. Poisson problem. Maximal absolute values in right hand side, triangular method, the whole boundary jets are fixed.

True solution: $x^{7}-21 x^{5} y^{2}+35 x^{3} y^{4}-7 x y^{6}+\sin 4 x y$.

\begin{tabular}{|c|c|c|c|c|c|c|c|}
\multicolumn{4}{c}{ without multiscaling } & \multicolumn{4}{c|}{ with multiscaling } \\
\hline $\mathrm{N} \backslash \mathrm{k}$ & 0 & 1 & 2 & & 0 & 1 & 2 \\
\hline 3 & 0.39 & 1.3 & 2.2 & & 0.39 & 1.3 & 6.2 \\
\hline 5 & 1.2 & 6.8 & 38.3 & & 1.2 & 3.7 & 15.6 \\
\hline 9 & 2.1 & 15.7 & 57.5 & & 2.1 & 6.6 & 37.9 \\
\hline 17 & 2.8 & 21.0 & 73.4 & & 2.8 & 7.55 & $\star$ \\
\hline
\end{tabular}


TABle 6.15. Poisson problem. Maximal absolute values in right hand side, triangular method, the whole boundary jets are fixed. True solution: $e^{3 x} \sin 3 y+\sin 4 x y$.

\begin{tabular}{l}
\multicolumn{4}{c}{ without multiscaling } & \multicolumn{4}{c|}{ with multiscaling } \\
\begin{tabular}{|c|c|c|c|c|c|c|c|}
\hline $\mathrm{N} \backslash \mathrm{k}$ & 0 & 1 & 2 & & 0 & 1 & 2 \\
\hline 3 & 6.3 & 17.5 & 46.4 & & 6.3 & 17.5 & 6.7 \\
\hline 5 & 5.4 & 19.9 & 56.4 & & 5.4 & 15.2 & 15.42 \\
\hline 9 & 5.3 & 26.3 & 75.2 & & 5.3 & 14.9 & 37.9 \\
\hline 17 & 5.3 & 29.2 & 83.9 & & 5.3 & 14.8 & $\star$ \\
\hline
\end{tabular}
\end{tabular}

TABle 6.16. Poisson problem. Maximal absolute values in right hand side, full method, the whole boundary jets are fixed. True solution: $x^{7}-21 x^{5} y^{2}+35 x^{3} y^{4}-7 x y^{6}+\sin 4 x y$.

\begin{tabular}{|c|c|c|c|c|c|c|c|}
\hline \multicolumn{4}{c}{ without multiscaling } & \multicolumn{4}{c|}{ with multiscaling } \\
\hline $\mathrm{N} \backslash \mathrm{k}$ & 0 & 1 & 2 & & 0 & 1 & 2 \\
\hline 3 & 0.39 & 1.9 & 8.6 & & 0.39 & 3.9 & 2.9 \\
\hline 5 & 1.2 & 10.1 & 246.2 & & 1.2 & 5.7 & 13.0 \\
\hline 9 & 2.1 & 28.9 & 2282. & & 2.1 & 16.2 & 55.3 \\
\hline 17 & 2.8 & 66. & 13220. & & 2.8 & 25.7 & $\star$ \\
\hline
\end{tabular}

TABLE 6.17. Poisson problem. Maximal absolute values in right hand side, full method, the whole boundary jets are fixed. True solution: $e^{3 x} \sin 3 y+\sin 4 x y$.

\begin{tabular}{|c|c|c|c|c|c|c|c|}
\multicolumn{4}{c}{ without multiscaling } & \multicolumn{4}{c|}{ with multiscaling } \\
\hline $\mathrm{N} \backslash \mathrm{k}$ & 0 & 1 & 2 & & 0 & 1 & 2 \\
\hline 3 & 6.2 & 14.4 & 36.9 & & 6.2 & 17.6 & 2.76 \\
\hline 5 & 5.4 & 42.5 & 2054. & & 5.4 & 34.9 & 12.3 \\
\hline 9 & 5.3 & 94.6 & 10261. & & 5.3 & 48.5 & 55.3 \\
\hline 17 & 5.3 & 200. & 45500 & & 5.3 & 54.7 & $\star$ \\
\hline
\end{tabular}

TABLE 6.18. Condition numbers, triangular method, the whole boundary jets are fixed.

\begin{tabular}{|c|c|c|c|c|c|c|c|}
\hline \multicolumn{4}{c|}{ without multiscaling } & \multicolumn{4}{c|}{ with multiscaling } \\
\hline $\mathrm{N} \backslash \mathrm{k}$ & 0 & 1 & 2 & & 0 & 1 & 2 \\
\hline 3 & 1 & 1 & 1 & & 1 & 1 & 1 \\
\hline 5 & 5.83 & 5.83 & 5.84 & & 5.83 & 5.92 & 6. \\
\hline 9 & 25.27 & 25.3 & 25.32 & & 25.27 & 26.7 & 28.2 \\
\hline 17 & 103.1 & 103.2 & $\star$ & & 103.1 & 123.7 & $\star$ \\
\hline
\end{tabular}


TABLE 6.19. Condition numbers, full method, the whole boundary jets are fixed.

\begin{tabular}{|c|c|c|c|c|c|c|c|}
\multicolumn{4}{c}{ without multiscaling } & \multicolumn{4}{c|}{ with multiscaling } \\
\hline $\mathrm{N} \backslash \mathrm{k}$ & 0 & 1 & 2 & & 0 & 1 & 2 \\
\hline 3 & 1 & 1 & 1 & & 1 & 1 & 1 \\
\hline 5 & 5.83 & 105.5 & $3.710^{6}$ & & 5.83 & 14.7 & $1.5610^{4}$ \\
\hline 9 & 25.27 & 1341. & $\star$ & & 25.27 & 57.5 & $5.5610^{4}$ \\
\hline 17 & 103.1 & $1.2310^{4}$ & $\star$ & & 103.1 & 230 & $\star$ \\
\hline
\end{tabular}

TABLE 6.20. Dirichlet problem. Discretization errors in jet-form. Analytic solution: $x^{7}-21 x^{5} y^{2}+35 x^{3} y^{4}-7 x y^{6}$. Full method, true Dirichlet boundary data.

\begin{tabular}{|c|c|c|c|}
\hline $\mathrm{N} \backslash \mathrm{k}$ & 0 & 1 & 2 \\
\hline 3 & $\{0.547\}$ & $\{0,0.058,0.036\}$ & $\{0,0,0,0,0\}$ \\
\hline 5 & $\{0.220\}$ & $\left\{8.10^{-5}, 0.003,0.002\right\}$ & $\{0,0,0,0,0\}$ \\
\hline 9 & $\{0.059\}$ & $\left\{2.310^{-6}, 1.10^{-4}, 7.10^{-5}\right\}$ & $\{0,0,0,0,0\}$ \\
\hline 17 & $\{0.015\}$ & $\left\{4.10^{-8}, 3.10^{-6}, 2.710^{-6}\right\}$ & $\star$ \\
\hline
\end{tabular}

TABLE 6.21. Dirichlet problem. Discretization errors in jet-form. Analytic solution: $e^{3 x} \sin 3 y$. Full method, true Dirichlet boundary data.

\begin{tabular}{|c|c|c|c|}
\hline $\mathrm{N} \backslash \mathrm{k}$ & 0 & 1 & 2 \\
\hline 3 & $\{0.95\}$ & $\left\{2.10^{-3}, 6.710^{-3}, 0.05\right\}$ & $\left\{3.310^{-4}, 0.003,0.001,0.06,0.02\right\}$ \\
\hline 5 & $\{0.3\}$ & $\left\{1.510^{-4}, 5.510^{-3}, 1.10^{-3}\right\}$ & $\left\{3.210^{-7}, 3.110^{-6}, 1.210^{-6}, 2.310^{-4}, 1.210^{-4}\right\}$ \\
\hline 9 & $\{0.08\}$ & $\left\{5.110^{-6}, 2.10^{-4}, 3.810^{-5}\right\}$ & $\left\{4.110^{-10}, 1.310^{-8}, 3.210^{-9}, 1.610^{-6}, 1.310^{-6}\right\}$ \\
\hline 17 & $\{0.02\}$ & $\left\{1.110^{-7}, 9.110^{-6}, 1.10^{-6}\right\}$ & $\star$ \\
\hline
\end{tabular}

TABle 6.22. Condition numbers, full method, true Dirichlet boundary data.

\begin{tabular}{|c|c|c|c|}
\hline $\mathrm{N} \backslash \mathrm{k}$ & 0 & 1 & 2 \\
\hline 3 & 1 & 39.3 & 18196 \\
\hline 5 & 5.8 & 291.6 & $5.210^{5}$ \\
\hline 9 & 25.3 & 2136 & $\star$ \\
\hline 17 & 103. & $1.610^{4}$ & $\star$ \\
\hline
\end{tabular}




\section{REFERENCES}

[1] E. Bichuch, and Y. Yomdin, Numerical solution of parabolic equation by the method of high order discretization, The Weizmann Institute of Science, preprint (1994).

[2] M. Gromov, Partial Differential Relations, A Series of Modern Surveys in Mathematics 3 Folge, Band 9, Springer-Verlag, 1986. MR 91e:53047

[3] E. Kochavi, R. Segev, and Y. Yomdin, Numerical Solution of Field Problems by Nonconforming Taylor Discretization., Applied Mathematical Modeling 15 (1991), 152-157.

[4] E. Kochavi, R. Segev, and Y. Yomdin, Modified algorithms for nonconforming Taylor discretization, Computers and Structures 49 (6) (1993), 969-979.

[5] A. N. Kolmogorov, and V. M. Tikomoirov, $\epsilon$-entropy and $\epsilon$-capacity of sets in functional spaces, AMS Transl. 17 (2) (1961), 277-364. MR 23:A2031

[6] B. Malgrange, Ideals of differentiable functions, Tata Institute of Fundamental Research, Bombay and Oxford University press, 1966. MR 35:3446

[7] K. Niijima, A posteriory error bounds for piecewise linear approximate solutions of elliptic equations of monotone type, Math. Comp. 58 (1992), 549-560. MR 92g:65112

[8] P. J. M. Sonnemans, L. P. H. De Goey, and J. K. Nieuwenhuizen, Optimal Use of a Numerical Method for Solving Differential Equations Based on Taylor Series Expansions., Intern. J. for Numerical Methods in Engeneering 32 (1991), 471-499. MR 92d:65123

[9] A. M. Vinogradov, I. S. Krasilshik and V. V. Lychagin, Geometry of jet-spaces and nonlinear differential equations, Moscow, 1977 (Russian). MR 88m:58211

[10] H. Whitney, Analytic extensions of differentiable functions defined in closed sets, Trans. Amer. Math. Soc. 36 (1934), 63-89.

[11] Y. Yomdin, Y. Elihay, Flexible high order discretization, preprint.

[12] L. Collatz, Numerische Behandlung von Differentialgleichungen, Springer, 1951. MR 13:285f

Department of Theoretical Mathematics, The Weizmann Institute of Science, REHOVOT 76100, ISRAEL

E-mail address: mswiener@pluto.mscc.huji.ac.il

E-mail address: yomdin@wisdom.weizmann.ac.il 